\title{
UPPER BOUNDS FOR ERGODIC SUMS OF INFINITE MEASURE PRESERVING TRANSFORMATIONS
}

\author{
JON AARONSON ${ }^{1}$ AND MANFRED DENKER ${ }^{1,2}$
}

ABSTRACT. For certain conservative, ergodic, infinite measure preserving transformations $T$ we identify increasing functions $A$, for which

$$
\limsup _{n \rightarrow \infty} \frac{1}{A(n)} \sum_{k=1}^{n} f \circ T^{k}=\int_{X} f d \mu \quad \text { a.e. }
$$

holds for any nonnegative integrable function $f$. In particular the results apply to some Markov shifts and number-theoretic transformations, and include the other law of the iterated logarithm.

\section{INTRODUCTION}

We study the asymptotic behaviour of $S_{n}=\sum_{k=1}^{n} f\left(x_{k}\right)$ where $\left(x_{k}\right)_{k=1}^{\infty}$ denotes the forward orbit of some point $x$ under an ergodic measure preserving transformation $T$ of a $\sigma$-finite, nonatomic measure space $(X, \mathscr{B}, \mu)$ (that is $\left.x_{k}=T^{k}(x), x \in X\right)$, and $f$ denotes a nonnegative, integrable function with positive integral on $X .^{12}$

In case the measure space is finite, the asymptotic behaviour of $S_{n}$ is given by the Birkhoff ergodic theorem [11], $\lim _{n \rightarrow \infty} \frac{1}{n} S_{n}=\int_{X} f d \mu / \mu(X)$ for $\mu$-a.e. $x \in X$, and so we restrict attention to infinite measure spaces. We make the additional assumption that $S_{n} \rightarrow \infty$ for $\mu$-a.e. $x \in X$ and for every such $f$, i.e. that $T$ is conservative. In this case the Hopf ergodic theorem [11] states that

$$
\lim _{n \rightarrow \infty} \frac{\sum_{k=1}^{n} f\left(x_{k}\right)}{\sum_{k=1}^{n} g\left(x_{k}\right)}=\frac{\int_{X} f d \mu}{\int_{X} g d \mu} \quad \text { a.e. }
$$

whenever $f$ and $g$ are nonnegative functions on $X$ with positive integrals (denoted by $f, g \in L_{+}^{1}(\mu)$ ).

Owing to the infinity of the measure space, it is never possible to replace the denominators $\sum_{k=1}^{n} g\left(x_{k}\right)$ by constants [3], and, indeed if $b(n) \uparrow, b(n) / \downarrow 0$ then $\lim \inf S_{n} / b(n)=0, \infty$ a.e. We search for sequences $A(n)>0$ such that

$$
\limsup _{n \rightarrow \infty} S_{n} / A(n)=\int_{X} f d \mu \quad \text { a.e. }
$$

for some and hence all $f \in L_{+}^{1}(\mu)$.

Received by the editors January 30, 1989.

1980 Mathematics Subject Classification (1985 Revision). Primary 28D05.

${ }^{1}$ Research partially supported by Deutsche Forschungsgemeinschaft SFB 170, Göttingen.

${ }^{2}$ Research partially supported by the California Institute of Technology. 
There are transformations for which no such sequences exist [2, §2]. In this paper we give results identifying, for a natural class of transformations, sequences $A(n)$ satisfying (1). Using these, we find upper and lower class functions for the sums of nonnegative, cf. mixing (see $\S 5$ ) stationary random variables which are in the domain of attraction of a positive stable law.

This class of transformations includes all recurrent Markov shifts with regularly varying return sequences (see below), and the "number theoretical" transformations of [24], of which perhaps the simplest is the transformation of G. Boole: $x \mapsto x-1 / x$, preserving Lebesgue measure on $\mathbf{R}$ (see [6 and 18]).

Next, we proceed to define our class of transformations, after introducing some relevant notation. Let $T$ be a conservative, ergodic, measure preserving transformation (c.e.m.p.t.) of the infinite, $\sigma$-finite, nonatomic measure space $(X, \mathscr{B}, \mu)$. The operator $f \rightarrow f \circ T$ on $L^{\infty}(\mu)$ has a dual which preserves $L^{1}(\mu)$. We denote the restriction of this dual operator to $L^{1}(\mu)$ by $\widehat{T}$. Clearly, $\int_{X} f g \circ T d \mu=\int_{X} \widehat{T}(f) g d \mu$ for $f \in L^{1}(\mu)$ and $g \in L^{\infty}(\mu)$. A set $A \in \mathscr{B}$, $0<\mu(A)<\infty$ is called a Darling-Kac (D-K) set for $T$ [4,9] if there are constants $a_{n}$ such that

$$
\lim _{n \rightarrow \infty} \frac{1}{a_{n}} \sum_{k=1}^{n} \widehat{T}^{k} 1_{A}=\mu(A) \quad \text { uniformly on } A .
$$

If a c.e.m.p.t. has a D-K set, then $[4, \S 1]$

$$
\lim _{n \rightarrow \infty} \frac{1}{a_{n}} \sum_{k=1}^{n} \widehat{T}^{k} f=\int_{X} f d \mu \quad \text { a.e. }
$$

for all $f \in L^{1}(\mu)$. Hence the sequence $a_{n}$ depends only on $T$ and not on the D-K set $A$. It is denoted by $a_{n}=a_{n}(T)$ and called a return sequence for $T$. See [1] for a further discussion of return sequences.

In this paper we restrict attention to transformations, whose return sequences are regularly varying with index $0<\alpha<1$, that is $a_{n}(T)=n^{\alpha} h(n)$, where, by Karamata's theorem [23],

$$
h(t)=\exp \left(\eta(t)+\int_{c}^{t} \varepsilon(s) / s d s\right)
$$

where $c \geq 1, \eta(t) \rightarrow \eta_{0} \in \mathbf{R}$ and $\varepsilon(t) \rightarrow 0$ as $t \rightarrow \infty$. Since (2) depends only on the asymptotic growth rate of the return sequence for $T$, there is no loss of generality in assuming that $\eta \equiv \eta_{0}, c=0, \varepsilon(s)=0$ for $0<s<1$ and that $|\varepsilon(s)| \leq \delta \leq \alpha / 4$ for all $s \geq 1$. We shall always assume the existence of a Darling-Kac set of which the return time process (sequence of interarrival times) has one of the mixing properties defined in $\S 1$. These mixing properties are satisfied, if, for example, the return time process is $\psi$-mixing.

Our class of transformations includes important examples from probability and dynamics. 
If $T$ is a null recurrent Markov shift with discrete state state space, and $A$ is the event of a visit to a fixed state at a fixed time, then the return time process to $A$ is independent. It also follows [9] that $A$ is a D-K set for $T$. In particular, if $T$ is the Markov shift of a centered random walk on the integers with finite jump variance $\sigma^{2}$ and the invariant measure is normalized, so that the events of visits to fixed states at fixed times have unit measure, then, by the local limit theorem,

$$
a_{n}(T) \sim \sqrt{\frac{2}{\pi \sigma^{2}}} n^{\frac{1}{2}} .
$$

If $T$ is a transformation satisfying the assumptions of $M$. Thaler [24] then [4, Theorem 3] $T$ has D-K sets, whose return time processes are mixing in the above sense. This is shown in the proof of Theorem 3 in [4]. The return sequence can also be calculated by this theorem. In particular, if $T$ is Boole's transformation $x \mapsto 1-1 / x$ preserving Lebesgue measure on $\mathbf{R}$, then

$$
a_{n}(T) \sim \frac{\sqrt{2}}{\pi} n^{\frac{1}{2}}
$$

Alternatively, if $T:[0,1] \rightarrow[0,1]$ is defined by

$$
T(x)=\left\{\frac{x}{\left(1-x^{1 / \alpha}\right)^{\alpha}}\right\},
$$

where $0<\alpha \leq 1$ and \{\} denotes the fractional part, then there is an absolutely continuous invariant measure with density $x^{-1 / \alpha} f(x)$, where $f$ is continuous and positive on $[0,1]$. The return sequence satisfies $a_{n}(T) \sim c n^{\alpha}$, when $0<$ $\alpha<1$, and $a_{n}(T) \sim n / \log n$, when $\alpha=1$.

For transformations $T$ of the class defined above, we identify increasing sequences $A(n)$ satisfying (1) (Theorem 4), and this identification only depends on the return sequence for $T$. In particular,

$$
\limsup _{n \rightarrow \infty} \frac{1}{a\left(n / L_{2}(n)\right) L_{2}(n)} \sum_{k=1}^{n} f \circ T^{k}=K_{\alpha} \int_{X} f d \mu \quad \text { a.e. },
$$

for any $f \in L_{+}^{1}(\mu)$, where $a_{n}(T) \sim a(n)=n^{\alpha} h(n), h$ slowly varying, and where $K_{\alpha}=\frac{\Gamma(1+\alpha)}{\alpha^{\alpha}(1-\alpha)^{1-\alpha}}$. Here and throughout $L(x)=L_{1}(x)=\log _{e} x$ and $L_{k+1}(x)=L\left(L_{k}(x)\right)$. These results hold simultaneously for similar transformations [1] (see the example at the end of $\S 4$ ).

Theorem 4 seems to be the first of its kind for transformations arising in dynamics, while the probabilistic examples have been considered before. This theorem was proved by K. L. Chung and G. A. Hunt [8] for $T$ being the Markov shift of the simple random walk on the integers, by M. Lipschutz [19] and N. Jain and W. Pruitt [14] for Markov shifts of more general random walks, the latter using an invariance principle and the other law of the iterated logarithm for stable processes [10, 17]. 
We apply the results of $\S 4$ to obtain results (Theorem 5 and Corollary 3 ) on upper and lower class functions for the sums of positive, stationary cf. mixing random variables which are in the domain of attraction of a stable law of fractional index. This is done in $\S 5$. Let $\left(X_{n}\right)_{n=1}^{\infty}$ be such a sequence of such random variables satisfying

$$
\operatorname{Prob}\left(X_{1} \geq t\right) \sim \frac{1}{\Gamma(1-\alpha) \Gamma(1+\alpha) t^{\alpha} h(t)} \quad \text { as } t \rightarrow \infty
$$

where $0<\alpha<1$ and where $h$ is slowly varying. Define $b(n)$ by $b(n)=$ $n^{1 / \alpha} h(b(n))^{-1 / \alpha}$. It is known (see [4] and references therein) that $\sum_{k=1}^{n} X_{k} / b(n)$ tends in distribution to a positive stable law of index $\alpha$. We show in Theorem 5 (c) that

$$
\liminf _{n \rightarrow \infty} \frac{1}{b\left(n / L_{2}(n)\right) L_{2}(n)} \sum_{k=1}^{n} X_{k}=\left(\frac{1}{K_{\alpha}}\right)^{\frac{1}{\alpha}} \quad \text { a.e. }
$$

The paper of M. Lipschutz [20] contains Corollary 3 in the independent case, however under severe additional assumptions on the slowly varying function $h$ and the paper of M. Wichura [25] contains a result implying Theorem 5(c) in the independent case.

To conclude this introduction, we explain our plan of attack. To this end let $T$ be a transformation of our class, $A$ be a suitable Darling-Kac set for $T$ and $S_{n}=\sum_{k=1}^{n} 1_{A} \circ T^{k}$. To prove our results, we shall use a new Borel-Cantelli lemma for sets of the form $A \cap\left\{S_{n} \geq t\right\}$, and an estimation of the measure of these sets.

The Borel-Cantelli lemma is proved in $\S 1$ (Theorem 1). Its proof uses heavily the structure of the set $\left\{S_{n} \geq t\right\}$ and needs only the mixing structure of the return time process to $A$. It is different from the corresponding result of $\mathrm{K}$. L. Chung and P. Erdös [7], which is used in [20].

The estimations of $\mu\left(A \cap\left\{S_{n} \geq t\right\}\right)$ (Theorem 3) are carried out in $\S 2$ and $\S 3$.

The method of proof relies on sharp estimations of $\int_{A} S_{n}^{p} d \mu$ (Theorem 2) for moderate $p$ 's, which are obtained using the dual operator $\widehat{T}$.

\section{Borel-Cantelli lemmas}

Let $(X, \mathscr{B}, \mu, T)$ be a conservative, ergodic measure preserving transformation, $A \in \mathscr{B}$ and $\mu(A)=1$. (If $0<\mu(A)<\infty$, then the measure $\mu$ can be renormalized so that $\mu(A)=1$, and the sequences $A(n)$ in (1) renormalized accordingly.) Let $\varphi$ be the first return time function to $A$ of points in $A$ :

$$
\varphi(x)=\min \left\{n \geq 1: T^{n}(x) \in A\right\} \quad(x \in A),
$$

$T_{A}: A \rightarrow A$ be the transformation induced by $T$ on $A[16]:$

$$
T_{A}(x)=T^{\varphi(x)}(x)
$$


$\varphi_{n}(x)$ be the time of the $n$th return of $x$ to $A$ under $T$ :

$$
\varphi_{n}(x)=\sum_{k=1}^{n} \varphi\left(T_{A}^{k-1}(x)\right),
$$

and

$$
S_{n}(x)=\sum_{k=1}^{n} 1_{A}\left(T^{k}(x)\right)
$$

the number of returns to $A$ up to time $n$. Clearly,

$$
S_{\varphi_{n}(x)}(x)=n \text { and } S_{n}(x) \geq m \Leftrightarrow \varphi_{m}(x) \leq n .
$$

In this section, we prove Borel-Cantelli lemmas for events of the form $A \cap$ $\left\{S_{K_{n}} \geq a_{n}\right\}$ where $K_{n}=\left[\gamma^{n}\right], \gamma>1$ and $a_{n}>0$, under certain conditions. The probabilities of these events will be estimated in the sequel, whence the identification of upper bound sequences.

Definition. Let $\mathscr{B}_{0}=\sigma\left\{\varphi \circ T_{A}^{k}: k \geq 0\right\}$.

(a) The return time process of $T$ on $A$ is said to be uniformly mixing from below (u.m.b.) if, for all $C \in \mathscr{B}_{0}$ with $\mu(C)>0$ there exist $\delta>0$ and $k_{0} \geq 1$ such that for all $n \geq 1, B \in \sigma\left(\left\{\varphi \circ T_{A}^{j}: 0 \leq j<n\right\}\right)$ and $k \geq k_{0}$ :

$$
\mu\left(B \cap T_{A}^{-(n+k)} C\right) \geq \delta \mu(B) .
$$

(b) The return time process of $T$ on $A$ is said to be strongly mixing from below (s.m.b.) if for any $C \in \mathscr{B}_{0}$ with $\mu(C)>0$, there exists $\alpha(n) \downarrow 0$ such that $\sum_{n=1}^{\infty} \alpha(n) / n<\infty$ and for all $n \geq 1, B \in \sigma\left(\left\{\varphi \circ T_{A}^{j}: 0 \leq j<n\right\}\right)$, and $k \geq 1$

$$
\mu\left(B \cap T_{A}^{-(n+k)} C\right) \geq \mu(B) \mu(C)-\alpha(k) .
$$

We prove first

Theorem 1. Fix $\gamma>1$, and let $K_{n}=\left[\gamma^{n}\right]$. Suppose that $a(n)>0$ is such that for all $M>0$ there exists $m \geq 1$ such that $a(n+m) \geq M a(n)$ for all $n \geq 1$. Suppose also that the return time process on $A$ is either (a) u.m.b. or (b) s.m.b. Then:

(i) if $\sum_{n=1}^{\infty} \mu\left(A \cap\left\{S_{K_{n}} \geq \beta a(n)\right\}\right)<\infty$ for all $\beta>1$ then

$$
\limsup _{n \rightarrow \infty} \frac{1}{a(n)} \sum_{k=1}^{K_{n}} f \circ T^{k} \leq \int_{X} f d \mu
$$

a.e. for any $f \in L_{+}^{1}$.

(ii) if $\sum_{n=1}^{\infty} \mu\left(A \cap\left\{S_{K_{n}} \geq r a(n)\right\}\right)=\infty$ for all $r<1$ then

$$
\limsup _{n \rightarrow \infty} \frac{1}{a(n)} \sum_{k=1}^{K_{n}} f \circ T^{k} \geq \int_{X} f d \mu
$$

a.e. for any $f \in L_{+}^{1}$. 
Remark 1. If there exists an $m$ such that $a(n+m) \geq 2 a(n)$ for $n \geq 1$ then $a(k m+t) \geq 2^{k} a(t)$ and hence $a(n) \geq \varepsilon 2^{[n / m]-1}$ where $\varepsilon=\min \{a(t): 4 \leq t \leq$ $m\}$. Thus there exists $\gamma_{1}>1$ such that $a(n) \geq \gamma_{1}^{n}$ for all large $n\left(\gamma_{1}<2^{1 / m}\right)$. Proof. (i) If $\sum_{n=1}^{\infty} \mu\left(A \cap\left\{S_{K_{n}} \geq \beta a(n)\right\}\right)<\infty$ for all $\beta>1$, then clearly $\limsup S_{K_{n}} / a(n) \leq 1$ a.e. on $A$, whence by ergodicity $\lim \sup S_{K_{n}} / a(n) \leq 1$ a.e. on $X$. By Hopf's ergodic theorem for every $f \in L_{+}^{1}$

$$
\lim \sup \frac{1}{a(n)} \sum_{k=1}^{K_{n}} f \circ T^{k} \leq \int_{X} f d \mu \quad \text { a.e. }
$$

(ii) Now suppose $\sum_{n=1}^{\infty} \mu\left(A \cap\left\{S_{K_{n}} \geq r a(n)\right\}\right)=\infty$ for all $r<1$. We prove that $\limsup _{n \rightarrow \infty} S_{K_{n}} / a(n) \geq 1$, which suffices by Hopf's ergodic theorem.

Fix $\varepsilon>0$. Let $m$ be an integer such that $a(m+n) \geq 4 a(n) \varepsilon^{-1}$. Let

$$
\begin{gathered}
D_{N}=\bigcap_{\nu=N}^{\infty} A \cap\left\{S_{K_{\nu}} \leq(1-\varepsilon) a(n)\right\} \\
A_{n}=A \cap\left\{S_{K_{n}} \geq\left(1-\frac{1}{2} \varepsilon\right) a(n)\right\}
\end{gathered}
$$

and

$$
B_{n}^{(N)}=A_{n} \cap T_{A}^{-[a(n)]} D_{N} .
$$

We must show that $\mu\left(D_{N}\right)=0$ for all $N \geq 1$.

Step 1. We show that $\sum_{n=1}^{\infty} 1_{B_{n}^{(N)}}(x) \leq N+m$ a.e. on $A$ for all $N \geq 1$.

In order to prove this, fix $N \geq 1$. For $x \in A$, let

$$
\nu_{0}=\nu_{0}(x)=\min \left\{\nu \geq N: x \in B_{\nu}^{(N)}\right\} \leq \infty .
$$

If $\nu_{0}(x)=\infty$ then there is no $\nu \geq N$ such that $x \in B_{\nu}^{(N)}$ and Step 1 is established.

We assume henceforth that $\nu_{0}(x)<\infty$, and that $x \in \limsup T^{-n} A$ ( $\supset A \bmod \mu$ by conservativity). For $\nu \geq \nu_{0}(x)+m$ :

$$
\begin{aligned}
S_{K_{\nu}}(x) & =S_{\varphi_{\left[a\left(\nu_{0}\right)\right]}(x)}(x)+S_{K_{\nu}-\varphi_{\left[a\left(\nu_{0}\right)\right]}(x)}\left(T^{\varphi_{\left[a\left(\nu_{0}\right)\right]}(x)}(x)\right) \\
& =\left[a\left(\nu_{0}\right)\right]+S_{K_{\nu}-\varphi_{\left[a\left(\nu_{0}\right)\right]}(x)}\left(T_{A}^{\left[a\left(\nu_{0}\right)\right]}(x)\right) \\
& \leq a\left(\nu_{0}\right)+S_{K_{\nu}}\left(T_{A}^{\left[a\left(\nu_{0}\right)\right]}(x)\right) \\
& \leq a\left(\nu_{0}\right)+(1-\varepsilon) a(\nu) \leq \frac{\varepsilon}{4} a(\nu)+(1-\varepsilon) a(\nu) \\
& =\left(1-\frac{3}{4} \varepsilon\right) a(\nu)<\left(1-\frac{\varepsilon}{2}\right) a(\nu),
\end{aligned}
$$

where we used that $x \in B_{\nu_{0}}^{(N)}$ implies that $T_{A}^{\left[a\left(\nu_{0}\right)\right]}(x) \in D_{N} \subseteq\left\{S_{K_{\nu}} \leq\right.$ $(1-\varepsilon) a(\nu)\}$ for every $\nu \geq N$. Hence $\nu_{0}(x)<\infty$ implies that $x \notin$ 
$A_{\nu}\left(\forall \nu \geq \nu_{0}+m\right)$ and therefore $x \notin B_{\nu}^{(N)}\left(\forall \nu \geq \nu_{0}+m\right)$. Thus

$$
\begin{aligned}
\sum_{\nu=1}^{\infty} 1_{B_{\nu}^{(N)}}(x)= & \sum_{\nu=1}^{N} 1_{B_{\nu}^{(N)}}(x)+\sum_{\nu=N+1}^{\nu_{0}(x)-1} 1_{B_{\nu}^{(N)}}(x)+\sum_{\nu=\nu_{0}(x)}^{\nu_{0}(x)+m-1} 1_{B_{\nu}^{(N)}}(x) \\
& +\sum_{\nu=\nu_{0}(x)+m}^{\infty} 1_{B_{\nu}^{(N)}}(x) \leq N+m .
\end{aligned}
$$

Step 2. We show finally that for every $N \geq 1 \mu\left(D_{N}\right)=0$.

We prove Step 2 by showing that if $\mu\left(D_{N}\right)>0$ for some $N \geq 1$ then $\sum_{n=1}^{\infty} \mu\left(B_{n}^{(N)}\right)=\infty$, in contradiction to Step 1. We give different proofs of this statement according to the assumptions made.

Under assumption (a), if $\mu\left(D_{N}\right)>0$ then there exist $\delta>0$ and $k_{0} \geq 1$ such that $\mu\left(B \cap T_{A}^{-(n+k)} D_{N}\right) \geq \delta \mu(B)$ whenever $k \geq k_{0}, n \geq 1$ and $B \in$ $\sigma\left(\left\{\varphi \circ T_{A}^{j}: 0 \leq j \leq n-1\right\}\right)$. Now

$$
\begin{aligned}
A_{\nu} & =\left\{S_{K_{\nu}} \geq\left(1-\frac{\varepsilon}{2}\right) a(\nu)\right\}=\left\{\varphi_{\left[\left(1-\frac{\varepsilon}{2}\right) a(\nu)\right]} \leq K_{\nu}\right\} \\
& \in \sigma\left(\left\{\varphi \circ T_{A}^{j}: 0 \leq j \leq\left[\left(1-\frac{\varepsilon}{2}\right) a(\nu)\right]-1\right\}\right) .
\end{aligned}
$$

Moreover there exists $\nu_{1}$ such that for all $\nu \geq \nu_{1}[a(\nu)]-\left[\left(1-\frac{\varepsilon}{2}\right) a(\nu)\right] \geq k_{0}$, whence for all $\nu \geq \nu_{1}$ we obtain

$$
\mu\left(B_{\nu}^{(N)}\right)=\mu\left(A_{\nu} \cap T_{A}^{-[a(\nu)]} D_{N}\right) \geq \delta \mu\left(A_{\nu}\right)
$$

and $\sum_{\nu=1}^{\infty} \mu\left(B_{\nu}^{(N)}\right)=\infty$.

Under assumption (b), writing $p_{\nu}=[a(\nu)]-\left[\left(1-\frac{\varepsilon}{2}\right) a(\nu)\right]$ we have that

$$
\mu\left(B_{\nu}^{(N)}\right)=\mu\left(A_{\nu} \cap T_{A}^{-[a(\nu)]} D_{N}\right) \geq \mu\left(A_{\nu}\right) \mu\left(D_{N}\right)-\alpha\left(p_{\nu}\right) .
$$

Now $p_{\nu} \sim \frac{\varepsilon}{2} a(\nu)$. By Remark 1, there exists $\gamma_{1} \in(1, \gamma)$ such that $a(\nu) \geq \gamma_{1}^{\nu}$ for $\nu$ large, whence for some $\gamma_{2} \in\left(1, \gamma_{1}\right.$ ] and some $\nu_{2}$ we have that $p_{\nu} \geq$ $\gamma_{2}^{\nu} \quad\left(\forall \nu \geq \nu_{2}\right)$ and $\sum_{\nu=1}^{\infty} \alpha\left(p_{\nu}\right) \leq \sum_{\nu=1}^{\infty} \alpha\left(\gamma_{2}^{\nu}\right)<\infty$ by condensation. Thus for some $M$

$$
\begin{aligned}
\sum_{\nu=1}^{\infty} \mu\left(B_{\nu}^{(N)}\right) & \geq \mu\left(D_{N}\right) \sum_{\nu=1}^{\infty} \mu\left(A_{\nu}\right)-\sum_{\nu=1}^{\infty} \alpha\left(p_{\nu}\right) \\
& \geq \mu\left(D_{N}\right) \sum_{\nu=1}^{\infty} \mu\left(A_{\nu}\right)-M=\infty
\end{aligned}
$$

if $\mu\left(D_{N}\right)>0$.

Corollary 1. Suppose that $b(n) \uparrow, \quad b(n) / n \downarrow$ as $n \uparrow$, and that for all $M>1$ there exists an $m \geq 1$ such that $b(m n) \geq M b(n)$ for all $n \geq 1$. Suppose also that the return time process of $T$ on $A$ is either (a) u.m.b. or (b) s.m.b. Then

(i) if $\sum_{n=1}^{\infty} \frac{1}{n} \mu\left(A \cap\left\{S_{n} \geq \beta b(n)\right\}\right)<\infty$ for all $\beta>1$, then

$$
\limsup _{n \rightarrow \infty} \frac{1}{b(n)} \sum_{k=1}^{n} f \circ T^{k} \leq \int_{X} f d \mu \text { a.e. }
$$

for every $f \in L_{+}^{1}$. 
(ii) if $\sum_{n=1}^{\infty} \frac{1}{n} \mu\left(A \cap\left\{S_{n} \geq r b(n)\right\}\right)=\infty$ for all $r<1$, then

$$
\limsup _{n \rightarrow \infty} \frac{1}{b(n)} \sum_{k=1}^{n} f \circ T^{k} \geq \int_{X} f d \mu \text { a.e. }
$$

for all $f \in L_{+}^{1}$.

Proof. For $\gamma>1$, let $K_{n}=K_{n}(\gamma)=\left[\gamma^{n}\right]$. Choose $\varepsilon=\varepsilon(\gamma)>0$ such that $\varepsilon \leq \sum_{k=K_{n}+1}^{K_{n+1}} k^{-1} \leq \varepsilon^{-1} \quad(\forall n \geq 1)$. Thus

$$
\begin{aligned}
\sum_{n=1}^{\infty} \frac{1}{n} \mu\left(A \cap\left\{S_{n} \geq \beta b(n)\right\}\right) & \geq \varepsilon \sum_{n=0}^{\infty} \mu\left(A \cap\left\{S_{K_{n}} \geq \beta b\left(K_{n+1}\right)\right\}\right) \\
& \geq \varepsilon \sum_{n=0}^{\infty} \mu\left(A \cap\left\{S_{K_{n}} \geq \beta K_{n+1} K_{n}^{-1} b\left(K_{n}\right)\right\}\right)
\end{aligned}
$$

since $b(n) / n \downarrow$ as $n \uparrow$. Since $\lim _{n \rightarrow \infty} K_{n+1} K_{n}^{-1}=\gamma$, we have that

$$
\begin{aligned}
& \sum_{n=1}^{\infty} \frac{1}{n} \mu\left(A \cap\left\{S_{n} \geq \beta b(n)\right\}\right)<\infty \quad(\forall \beta>1) \\
& \quad \Rightarrow \sum_{n=1}^{\infty} \mu\left(A \cap\left\{S_{K_{n}(\gamma)} \geq \beta \gamma b\left(K_{n}(\gamma)\right)\right\}\right)<\infty \quad(\forall \beta, \gamma>1) .
\end{aligned}
$$

Similarly,

$$
\begin{aligned}
& \sum_{n=1}^{\infty} \frac{1}{n} \mu\left(A \cap\left\{S_{n} \geq r b(n)\right\}\right)=\infty \quad(\forall r<1) \\
& \quad \Rightarrow \sum_{n=1}^{\infty} \mu\left(A \cap\left\{S_{K_{n}(\gamma)} \geq r \gamma^{-1} b\left(K_{n}(\gamma)\right)\right\}\right)=\infty \quad(\forall r<1, \gamma>1) .
\end{aligned}
$$

Now fix $\gamma>1$ and let $a(n)=b\left(K_{n}(\gamma)\right)$. The assumptions of Theorem 1 are satisfied, and so

(i) if $\sum_{n=1}^{\infty} \frac{1}{n} \mu\left(A \cap\left\{S_{n} \geq \beta b(n)\right\}\right)<\infty \quad(\forall \beta>1)$, then

$$
\limsup _{n \rightarrow \infty} \frac{S_{K_{n}}}{a(n)} \leq \gamma \text { a.e. }
$$

whence

$$
\limsup _{n \rightarrow \infty} \frac{S_{n}}{b(n)} \leq \gamma \limsup _{n \rightarrow \infty} \frac{a\left(K_{n+1}\right)}{a\left(K_{n}\right)} \leq \gamma^{2} \text { a.e. }
$$

(ii) if $\sum_{n=1}^{\infty} \frac{1}{n} \mu\left(A \cap\left\{S_{n} \geq r b(n)\right\}\right)=\infty \quad(\forall r<1)$, then

$$
\limsup _{n \rightarrow \infty} \frac{S_{K_{n}}}{a(n)} \geq \gamma^{-1}
$$

whence

$$
\limsup _{n \rightarrow \infty} \frac{S_{n}}{b(n)} \geq \limsup _{n \rightarrow \infty} \frac{S_{K_{n}}}{a(n)} \geq \gamma^{-1} \text { a.e. }
$$


Letting $\gamma \downarrow 1$ the corollary follows from Hopf's ergodic theorem.

\section{ESTIMATION OF MOMENTS}

Suppose that $(X, \mathscr{B}, \mu, T)$ is a conservative and ergodic measure preserving transformation and $a_{n}(T) \sim n^{\alpha} h(n)$ where $0<\alpha \leq 1$ and (as in the introduction) $\log h(t)=\eta_{0}+\int_{0}^{t} \varepsilon(s) / s d s$ where

$$
\begin{aligned}
& \varepsilon(s)=0 \text { for } 0<s<1, \\
& \lim _{s \rightarrow \infty} \varepsilon(s)=0 \\
& |\varepsilon(s)| \leq \delta<\frac{1}{4} \alpha \quad(\forall s>0) .
\end{aligned}
$$

Suppose that $A \in \mathscr{B}$ is a Darling-Kac set for $T$ with $\mu(A)=1$. If $0<$ $\mu(A)<\infty$ we can renormalize the measure $\mu$ and the return sequence $a_{n}(T)$ and the sequences $A(n)$ in $(1)$, so that $\mu(A)=1$. Let $S_{n}=\sum_{k=1}^{n} 1_{A} \circ T^{k}$. In this section we estimate the $p$ th moments of $S_{n}$ from above and below.

Theorem 2. For every $\beta>1$ there exists $n_{\beta} \in \mathbf{N}$ such that for all integers $n \geq n_{\beta}$ and $n_{\beta} \leq p \leq L_{2}(n)^{2}$,

$$
\left(\int_{A} S_{n}^{p} d \mu\right)^{1 / p}=\theta(n, p) \frac{\Gamma(1+\alpha)}{\alpha^{\alpha} e^{(1-\alpha)}} p^{(1-\alpha)} n^{\alpha} h(n / p)
$$

where $\theta(n, p) \in\left(\beta^{-1}, \beta\right)$.

Remark. (a) In case that

$$
\sup \left\{|\varepsilon(t)-\varepsilon(s)|: \frac{t}{L_{2}(t)} \leq s \leq t\right\}=o\left(\frac{1}{L_{3}(t)}\right) \quad \text { as } t \rightarrow \infty
$$

we have

$$
\left(\int_{A} S_{n}^{p} d \mu\right)^{1 / p}=\theta(n, p) \frac{\Gamma(1+\alpha)}{\alpha^{\alpha} e^{(1-\alpha)}} p^{(1-\alpha-\varepsilon(n))} n^{\alpha} h(n) .
$$

(b) In case

$$
\sup _{t \leq s \leq t L_{2}(t)}\left|\frac{h(s)}{h(t)}-1\right| \longrightarrow 0
$$

we have that

$$
\left(\int_{A} S_{n}^{p} d \mu\right)^{1 / p}=\theta(n, p) \frac{\Gamma(1+\alpha)}{\alpha^{\alpha} e^{(1-\alpha)}} p^{(1-\alpha)} n^{\alpha} h(n) .
$$

Proof. The proof uses a sequence of lemmas, one of which (Lemma 2.8) is the crucial one. When Lemmas 2.10, 2.11 and 2.12 are established, Theorem 2 follows immediately, using Stirling's formula.

Define $a(p, n): X \rightarrow \mathbf{Z}_{+}(n, p \in \mathbf{N})$ by

$$
a(0, n) \equiv 1, \quad a(p+1, n)(x)=\sum_{k=1}^{n} 1_{A}\left(T^{k}(x)\right) a(p, n-k)\left(T^{k}(x)\right)
$$

and $\gamma_{p}(q) \in \mathbf{N}$ by $\gamma_{1}(q)=\delta_{1, q}, \quad \gamma_{p+1}(q)=q\left(\gamma_{p}(q)+\gamma_{p}(q-1)\right)$. 
Lemma 2.1. For $n \geq p \geq 1$ we have

$$
S_{n}(x)^{p}=\sum_{q=1}^{p} \gamma_{p}(q) a(q, n)(x) .
$$

Proof. A direct computation shows

$$
S_{n} a(p, n)=(p+1) a(p+1, n)+p a(p, n) .
$$

This is done by changing the order of summation in

$$
S_{n} a(p, n)=S_{n} \sum \prod_{\nu=1}^{p} 1_{A} \circ T^{k_{\nu}}
$$

where $\sum$ extends over $1 \leq k_{1}<k_{2}<\cdots<k_{p} \leq n$. The formula then follows by induction.

Let $\bar{a}(p, n)=\int_{A} a(p, n) d \mu, u_{k}=\int_{k-1}^{k} \alpha s^{\alpha-1} h(s) d s$.

Lemma 2.2. There exist $\beta_{1}(k) \downarrow 1$ and $\beta_{-1}(k) \uparrow 1$ as $k \uparrow \infty$ such that for all $n, p \geq 1$

$$
\sum_{k=1}^{n} \beta_{-1}(k) u_{k} \bar{a}(p, n-k) \leq \bar{a}(p+1, n) \leq \sum_{k=1}^{n} \beta_{1}(k) u_{k} \bar{a}(p, n-k) .
$$

Proof. By Karamata's theorem, $a_{n}(T) \sim \sum_{k=1}^{n} u_{k}$ as $n \rightarrow \infty$. Thus, there are $\beta_{1}(k) \downarrow 1$ and $\beta_{-1}(k) \uparrow 1$ such that for all $n \geq 1$ and $x \in A$ :

$$
\beta_{-1}(n) \sum_{k=1}^{n} u_{k} \leq T_{n}(x) \leq \beta_{1}(n) \sum_{k=1}^{n} u_{k}
$$

where $T_{n}(x)=\sum_{k=1}^{n} \widehat{T}^{k} 1_{A}(x)$. Set $T_{0}(x) \equiv 0$.

Set $\bar{u}_{k}=\sum_{k=1}^{n} \beta_{1}(k) u_{k}, \quad \underline{u}_{n}=\sum_{k=1}^{n} \beta_{-1}(k) u_{k}$ and $\bar{u}_{0}=\underline{u}_{0} \equiv 0$. Then for $x \in A$ :

and

$$
\underline{u}_{n} \leq T_{n}(x) \leq \bar{u}_{n}
$$

$$
\begin{aligned}
\sum_{k=1}^{n} \widehat{T}^{k} 1_{A}(x) a(p, n-k)(x) & =\sum_{k=1}^{n}\left(T_{k}(x)-T_{k-1}(x)\right) a(p, n-k)(x) \\
& =\sum_{k=1}^{n} T_{k}(x)[a(p, n-k)(x)-a(p, n-k-1)(x)] .
\end{aligned}
$$

Since $a(p, n)(x) \geq a(p, n-1)(x)$ for $n \geq 1$, we have

$$
\begin{aligned}
& \sum_{k=1}^{n} \underline{u}_{k}[a(p, n-k)(x)-a(p, n-k-1)(x)] \\
& \leq \sum_{k=1}^{n} \widehat{T}^{k} 1_{A}(x) a(p, n-k)(x) \\
& \leq \sum_{k=1}^{n} \bar{u}_{k}[a(p, n-k)(x)-a(p, n-k-1)(x)] .
\end{aligned}
$$


Whence

$$
\begin{aligned}
\sum_{k=1}^{n} \beta_{-1}(k) u_{k} a(p, n-k)(x) & \leq \sum_{k=1}^{n} \widehat{T}^{k} 1_{A}(x) a(p, n-k)(x) \\
& \leq \sum_{k=1}^{n} \beta_{1}(k) u_{k} a(p, n-k)(x) .
\end{aligned}
$$

The lemma now follows by integrating these inequalities on $A$.

Note that $s^{\alpha-1} h(s) \downarrow$ as $s \uparrow, s>0$ by assumption on $h$. Set $\beta_{0}(s) \equiv 1(s>$ 0 ) and extend the domains of definition of $\beta_{-1}$ and $\beta_{1}$ by setting

$$
\begin{aligned}
& \beta_{1}(s)=\beta_{1}([s]+1) \quad(s>0, s \notin \mathbf{N}), \\
& \beta_{-1}(s)=\beta_{-1}([s]+1) \quad(s>0, s \notin \mathbf{N}) .
\end{aligned}
$$

Define

and

$$
A_{0}(0, t)=A_{-1}(0, t)=A_{1}(0, t) \equiv 1
$$

$$
\begin{aligned}
A_{i}(p+1, t) & =\int_{0}^{t} \beta_{i}(s) \alpha s^{\alpha-1} h(s) A_{i}(p, t-s) d s \\
& =\int_{0}^{t} \beta_{i}(t-s) \alpha(t-s)^{\alpha-1} h(t-s) A_{i}(p, s) d s \quad(i=0, \pm 1) .
\end{aligned}
$$

Lemma 2.3. For all $n, p \geq 1$

$$
A_{-1}(p, n) \leq \bar{a}(p, n) \leq A_{1}(p, n) .
$$

Proof. By Lemma 2.2 for all $n, p \geq 1$

$$
\begin{aligned}
& \int_{0}^{n} \beta_{-1}(s) \alpha s^{\alpha-1} h(s) \bar{a}(p,[n-s]) d s \\
& \quad \leq \bar{a}(p+1, n) \leq \int_{0}^{n} \beta_{1}(s) \alpha s^{\alpha-1} h(s) \bar{a}(p,[n-s]) d s .
\end{aligned}
$$

Lemma 2.3 follows by induction on $p$.

Clearly (by induction)

$$
A_{-1}(p, t) \leq A_{0}(p, t) \leq A_{1}(p, t) \quad(p \geq 0, t \geq 0) .
$$

Moreover $A_{1}(p, t) \leq \beta_{1}(0)^{p} A_{0}(p, t)$ and $A_{-1}(p, t) \geq \beta_{-1}(0)^{-p} A_{0}(p, t)$.

Now, let

$$
M_{i}(p, t)=\frac{\Gamma(1+\alpha p)}{\Gamma(1+\alpha)^{p} t^{\alpha p}} A_{i}(p, t) .
$$

It follows that $M_{i}(0, t) \equiv 1$ and

$$
M_{i}(p+1, t)=\frac{1}{C_{p}(\alpha)} \int_{0}^{1} u^{\alpha p} M_{i}(p, u t)(1-u)^{\alpha-1} h((1-u) t) \beta_{i}((1-u) t) d u
$$

where

$$
C_{p}(\alpha)=\frac{\Gamma(\alpha) \Gamma(1+\alpha p)}{\Gamma(1+\alpha(p+1))}=\int_{0}^{1}(1-u)^{\alpha-1} u^{\alpha p} d u=\frac{\Gamma(\alpha)}{\alpha^{\alpha} p^{\alpha}}\left(1+o_{p}(1)\right) .
$$

Clearly $M_{-1}(p, t) \leq M_{0}(p, t) \leq M_{1}(p, t)$. 
Our next task is to establish the asymptotic growth of $M_{i}(p, t)^{1 / p}$ as $t \rightarrow \infty$, $p \leq L_{2}(t)^{2}$, in order to prove Lemma 2.8 .

Lemma 2.4. There exists a constant $M$ such that for every $p \geq 0$ and $t>0$

$$
M_{1}(p, t) \leq\left(M p^{\delta} h(t)\right)^{p} .
$$

Proof. Assume $p \geq 0$ and that there is some constant $\bar{M}(p)<\infty$ such that $M_{1}(p, t) \leq \bar{M}(p) h(t)^{p}$. Using $h(u t) \leq u^{-\delta} h(t)$ for $t>0, u \in(0,1)$ it follows that

$$
\begin{aligned}
M_{1}(p+1, t) & \leq \frac{\beta_{1}(0) \bar{M}(p)}{C_{p}(\alpha)} h(t)^{p+1} \int_{0}^{1}(1-u)^{\alpha-1} \frac{h((1-u) t)}{h(t)}\left(u^{\alpha} \frac{h(u t)}{h(t)}\right)^{p} d u \\
& \leq \beta_{1}(0) C_{p}(\alpha)^{-1} \bar{M}(p) h(t)^{p+1} \int_{0}^{1}(1-u)^{\alpha-\delta-1} u^{(\alpha-\delta) p} d u \\
& =\beta_{1}(0) \bar{M}(p) h(t)^{p+1} \frac{C_{p}(\alpha-\delta)}{C_{p}(\alpha)} \\
& \leq \beta_{1}(0) C \bar{M}(p) h(t)^{p+1} p^{\delta}
\end{aligned}
$$

where the constant $C$ is chosen to satisfy $C_{p}(\alpha-\delta) / C_{p}(\alpha) \leq C p^{\delta}$ for every $p \geq 1$.

Since $M_{1}(0, t) \equiv 1$, it follows by induction that

$$
M_{1}(p, t) \leq\left(\beta_{1}(0) C h(t)\right)^{p}(p !)^{\delta} \leq\left(\beta_{1}(0) C h(t)\right)^{p} p^{\delta p} .
$$

Lemma 2.5. Suppose that for some constant $a>0$ and some $\tau>0 \inf _{t \geq \tau} \beta_{-1}(t)$ $\geq a$. Then there exist constants $M>0$ and $K \geq 2$ such that for every $p \geq 0$ and $t \geq K^{p} \tau$

$$
M_{-1}(p, t) \geq\left(M p^{\delta}\right)^{-p} h(t)^{p} .
$$

Proof. Assume $p \geq 0$ and that there is some constant $\underline{M}(p)>0$ such that $M_{-1}(p, t) \geq \underline{M}(p) h(t)^{p}$ for $t \geq K^{p} \tau$, where $K \geq 2$ will be specified in the sequel, so that the following estimations work. Using $h(u t) \geq u^{\delta} h(t)$ for $t>0$, $u \in(0,1)$,

$$
\begin{aligned}
& \frac{M_{-1}(p+1, t)}{h(t)^{p+1}} \geq \frac{a \underline{M}(p)}{C_{p}(\alpha)} \int_{K^{p} \tau / t}^{1-\tau / t}\left(u^{\alpha} \frac{h(u t)}{h(t)}\right)^{p}(1-u)^{\alpha-1} \frac{h((1-u) t)}{h(t)} d u \\
& \geq \frac{a \underline{M}(p)}{C_{p}(\alpha)} \int_{K^{p} \tau / t}^{1-\tau / t} u^{(\alpha+\delta) p}(1-u)^{\alpha+\delta-1} d u \\
& =\frac{a \underline{M}(p)}{C_{p}(\alpha)} C_{p}(\alpha+\delta)\left[1-\frac{1}{C_{p}(\alpha+\delta)} \int_{\left[0, K^{p} \tau / t\right] \cup[1-\tau / t, 1]} u^{(\alpha+\delta) p}(1-u)^{\alpha+\delta-1} d u\right] .
\end{aligned}
$$


Now, if $t \geq K^{p+1} \tau$,

$$
\begin{aligned}
\int_{1-\tau / t}^{1} u^{(\alpha+\delta) p}(1-u)^{\alpha+\delta-1} d u & =\int_{0}^{\tau / t}(1-u)^{(\alpha+\delta) p} u^{\alpha+\delta-1} d u \\
& \leq(\alpha+\delta)^{-1}(\tau / t)^{\alpha+\delta} \leq(\alpha+\delta)^{-1}\left(K^{-p-1}\right)^{\alpha+\delta} \\
& \leq(\alpha+\delta)^{-1} K^{-(p+1)(\alpha+\delta)}(p+1)^{\alpha+\delta} A C_{p}(\alpha+\delta),
\end{aligned}
$$

where the constant $A$ is chosen so that $(p+1)^{-(\alpha+\delta)} \leq A C_{p}(\alpha+\delta)$ for every $p \geq 0$. Similarly, for $t \geq K^{p+1} \tau$,

$$
\begin{aligned}
\int_{0}^{K^{p} \tau / t} u^{(\alpha+\delta) p}(1-u)^{\alpha+\delta-1} d u & \leq\left(1-K^{p} \tau / t\right)^{\alpha+\delta-1} \frac{1}{(\alpha+\delta) p+1}\left(K^{p} \tau / t\right)^{(\alpha+\delta) p+1} \\
& \leq(1-1 / K)^{\alpha+\delta-1} \frac{1}{(\alpha+\delta) p+1} K^{-(\alpha+\delta) p-1} \\
& \leq \frac{1}{\alpha+\delta} K^{-(\alpha+\delta)(p+1)} A C_{p}(\alpha+\delta)
\end{aligned}
$$

where $A$ is as before. Consequently, for $t \geq K^{p+1} \tau$,

$$
\frac{M_{-1}(p+1, t)}{h(t)^{p+1}} \geq \frac{a \underline{M}(p) C_{p}(\alpha+\delta)}{C_{p}(\alpha)}\left[1-\frac{A}{\alpha+\delta}\left(\frac{1}{K}\right)^{(\alpha+\delta)(p+1)}\left((p+1)^{\alpha+\delta}+1\right)\right] \text {. }
$$

Finally, choose $K$ so large that for all $p \geq 0$

$$
\frac{A}{\alpha+\delta}\left(\frac{1}{K}\right)^{(\alpha+\delta)(p+1)}\left((p+1)^{\alpha+\delta}+1\right)<\frac{1}{2}
$$

whence $M_{-1}(p+1, t) \geq \frac{a}{2} \underline{M}(p) C p^{-\delta} h(t)^{p+1}$ where the constant $C$ satisfies $C_{p}(\alpha+\delta) \geq C p^{-\delta} C_{p}(\alpha)$ for every $p \geq 1$. Note that

$$
\underline{M}(p)=\inf _{K^{p} \tau \leq s \leq t-\tau} M_{-1}(p, s) \beta_{-1}(t-s) h(s)^{-p}
$$

and hence, by induction, for $p \geq 0$ and $t \geq K^{p} \tau$

$$
M_{-1}(p, t) \geq\left(\frac{a C}{2}\right)^{p} p^{-\delta p} h(t)^{p}
$$

The last two lemmas are crude bounds of $M_{i}(p, t)$, which are needed for the finer estimations in the proofs of the following two lemmas.

Lemma 2.6. There exist constants $K$ and $m_{0}$, and for every $m \geq m_{0}$, there exists a constant $p_{0} \geq 2 m$ such that for any $p \geq p_{0}$ and $t>0$ we have

$$
M_{1}(p, t) \leq\left[\beta_{1}\left(\frac{t}{p !}\right)\right]^{p}\left[(m \exp (2 m))^{\delta_{p}(t)}+\frac{K}{m^{\alpha / 2}}\right]^{p} h(t / p)^{p}\left(M\left(2 p_{0}\right)^{2 \delta}\right)^{2 p_{0}},
$$

where $M$ is defined in Lemma 2.4 and where $\delta_{p}(t)=\sup _{s \geq t /(p !)}|\varepsilon(s)|$.

Proof. Define $K=\beta_{1}(0) e^{\delta}(\alpha-\delta)^{-1} A+2$ where $A$ is chosen so that $p^{-\alpha} \leq$ $A C_{p}(\alpha)$ for every $p \geq 1$, and let $m_{0}$ be so large, that for $m \geq m_{0}$ and $p \geq 2 m$

$$
2 \beta_{1}(0) m^{2 \alpha-1}\left(1-\frac{m}{p+1}\right)^{\alpha p / 2} \leq \alpha p^{\alpha} C_{p}(\alpha) \text {. }
$$


Let $m$ be given and choose $p_{0}$ so large that for $p \geq p_{0}$ $\beta_{1}(0)^{p+1}\left((m \exp (2 m))^{\delta}+K m^{-\alpha / 2}\right)^{p}(p+1)^{\delta}(p+1)^{-(\alpha-\delta) p} \leq(\alpha+\delta) m^{-\alpha / 2} C_{p}(\alpha)$.

We shall now prove the lemma by induction over $p \geq p_{0}$. Since $h(t) / h(t / p) \leq$ $p^{\delta}$, the lemma holds for $p=p_{0}$ by Lemma 2.4. Assume now it holds for $p \geq p_{0}$ and let $t>0$. Then, writing

$$
M(p, t)=\frac{M_{1}(p, t)}{h(t / p)^{p}\left(M\left(2 p_{0}\right)^{2 \delta}\right)^{2 p_{0}}}
$$

we have

$$
\begin{array}{r}
M(p+1, t)=C_{p}(\alpha)^{-1} \int_{0}^{1} u^{\alpha p}\left(\frac{h(u t / p)}{h(t /(p+1))}\right)^{p}(1-u)^{\alpha-1} \\
\times \frac{h((1-u) t)}{h(t /(p+1))} \beta_{1}((1-u) t) M(p, u t) d u .
\end{array}
$$

The integral on the right-hand side will be split into the sum of five integrals:

$$
I_{l}=\int_{A_{l}} u^{\alpha p}\left(\frac{h(u t / p)}{h(t /(p+1))}\right)^{p}(1-u)^{\alpha-1} \frac{h((1-u) t)}{h(t /(p+1))} \beta_{1}((1-u) t) M(p, u t) d u
$$

where $A_{1}=[0,1 /(p+1)], A_{2}=(1-1 /(m(p+1)), 1], A_{3}=(1 /(p+1), 1-$ $m /(p+1)], A_{4}=(1-m /(p+1), 1-1 /(p+1)]$ and $A_{5}=(1-1 /(p+1), 1-$ $1 /(m(p+1))]$.

Each of them will be estimated separately using

$$
\begin{aligned}
& \frac{h((1-u) t)}{h(t /(p+1))} \leq \begin{cases}(p+1)^{\delta_{p+1}(t)}(1-u)^{\delta_{p+1}(t)}, & \text { if } u \leq 1-\frac{1}{p+1} \\
(p+1)^{-\delta_{p+1}(t)}(1-u)^{-\delta_{p+1}(t)}, & \text { if } 1-\frac{1}{p+1} \leq u \leq 1-\frac{1}{m(p+1)} \\
(p+1)^{-\delta}(1-u)^{-\delta}, & \text { if } 1-\frac{1}{m(p+1)} \leq u \leq 1,\end{cases} \\
& \frac{h(u t / p)}{h(t /(p+1))} \leq \begin{cases}(1+1 / p)^{\delta_{p+1}(t)} u^{\delta_{p+1}(t)}, & \text { if } 1-\frac{1}{p+1} \leq u, \\
(1+1 / p)^{-\delta_{p+1}(t)} u^{-\delta_{p+1}(t)}, & \text { if } \frac{1}{p+1} \leq u \leq 1-\frac{1}{p+1} \\
(1+1 / p)^{-\delta} u^{-\delta}, & \text { if } 0 \leq u \leq \frac{1}{p+1}\end{cases}
\end{aligned}
$$

for the given $m$. It is important for the estimations of $M(p, u t)$ and $\beta_{1}((1-u) t)$ to note that for $u \geq 1 /(p+1)$ we have that $\delta_{p}(u t) \leq \delta_{p+1}(t)$ and that $\beta_{1}(u t /(p !)) \leq \beta_{1}(t /((p+1) !))$. 
Using the induction hypothesis for small values of $u$ we first note that

$$
\begin{aligned}
I_{1} \leq & \beta_{1}(0)^{p+1}\left((m \exp (2 m))^{\delta}+\frac{K}{m^{\alpha / 2}}\right)^{p} \\
& \times \int_{A_{1}} u^{(\alpha-\delta) p}(1+1 / p)^{-\delta p}(1-u)^{\alpha+\delta-1}(p+1)^{\delta} d u \\
\leq & \beta_{1}(0)^{p+1}\left((m \exp (2 m))^{\delta}+\frac{K}{m^{\alpha / 2}}\right)^{p} \frac{(p+1)^{\delta}}{(\alpha+\delta)(p+1)^{(\alpha-\delta) p}} \\
\leq & m^{-\alpha / 2} C_{p}(\alpha) .
\end{aligned}
$$

For the remaining integrals we apply the induction hypothesis to values of $u$ such that $\delta_{p}(u t) \leq \delta_{p+1}(t)$. We denote

$$
J_{l}=I_{l} \beta_{1}(t /(p+1) !)^{-p}\left((m \exp (2 m))^{\delta_{p+1}(t)}+K m^{-\alpha / 2}\right)^{-p},
$$

and obtain for the second integral

$$
\begin{aligned}
J_{2} & \leq \beta_{1}(0) \int_{A_{2}} u^{(\alpha+\delta) p}(1+1 / p)^{\delta p}(1-u)^{\alpha-\delta-1}(p+1)^{-\delta} d u \\
& =\beta_{1}(0)(p+1)^{-\delta}(1+1 / p)^{\delta p} \int_{0}^{1 / m(p+1)}(1-u)^{(\alpha+\delta) p} u^{\alpha-\delta-1} d u \\
& \leq \beta_{1}(0)(p+1)^{-\delta}(1+1 / p)^{\delta p}(m(p+1))^{-(\alpha-\delta)}(\alpha-\delta)^{-1} \\
& \leq A e^{\delta} \beta_{1}(0) \frac{C_{p}(\alpha)}{(\alpha-\delta) m^{\alpha-\delta}} \\
& =(K-2) m^{-\alpha / 2} C_{p}(\alpha),
\end{aligned}
$$

where $p^{-\alpha} \leq A C_{p}(\alpha)$ as before.

Since $\delta<\alpha / 4$, we have for $u \leq 1-m /(p+1)$

$$
u^{\left.\frac{\alpha}{2}-\delta_{p+1}(t)\right) p}(1-u)^{\delta_{p+1}(t)} \leq(1-m /(p+1))^{\left(\frac{\alpha}{2}-\delta_{p+1}(t)\right) p}(m /(p+1))^{\delta_{p+1}(t)} .
$$

Therefore

$$
\begin{aligned}
J_{3} \leq & \beta_{1}\left(\frac{t}{(p+1) !}\right) \frac{(p+1)^{\delta_{p+1}(t)}}{(1+1 / p)^{\delta_{p+1}(t) p}} \int_{A_{3}} u^{\left(\alpha-\delta_{p+1}(t)\right) p}(1-u)^{\alpha+\delta_{p+1}(t)-1} d u \\
\leq & \beta_{1}\left(\frac{t}{(p+1) !}\right)\left(\frac{m}{p+1}\right)^{\alpha+\delta_{p+1}(t)-1}(p+1)^{\delta_{p+1}(t)} \\
& \times\left(1-\frac{m}{p+1}\right)^{\left(\frac{\alpha}{2}-\delta_{p+1}(t)\right) p} \int_{A_{3}} u^{\alpha p / 2} d u \\
\leq & \beta_{1}\left(\frac{t}{(p+1) !}\right)\left(\frac{m}{p+1}\right)^{\alpha+\delta_{p+1}(t)-1}(p+1)^{\delta_{p+1}(t)} \frac{(1-m /(p+1))^{\left(\alpha-\delta_{p+1}(t)\right) p+1}}{1+\alpha p / 2} \\
\leq & \beta_{1}(0) 2 \alpha^{-1} m^{\frac{3}{2} \alpha-1} p^{-\alpha}(1-m /(p+1))^{\alpha p / 2} \\
\leq & m^{-\alpha / 2} C_{p}(\alpha) .
\end{aligned}
$$


Now

$$
\begin{aligned}
J_{4} & \leq \beta_{1}\left(\frac{t}{(p+1) !}\right) \frac{(p+1)^{\delta_{p+1}(t)}}{(1+1 / p)^{\delta_{p+1}(t) p}} \int_{A_{4}} u^{\left(\alpha-\delta_{p+1}(t)\right) p}(1-u)^{\alpha+\delta_{p+1}(t)-1} d u \\
& \leq \beta_{1}\left(\frac{t}{(p+1) !}\right)\left(\frac{(p+1) m /(p+1)}{(1-m /(p+1))^{p}}\right)^{\delta_{p+1}(t)} \int_{A_{4}} u^{\alpha p}(1-u)^{\alpha-1} d u \\
& \leq \beta_{1}\left(\frac{t}{(p+1) !}\right) e^{2 m \delta_{p+1}(t)} m^{\delta_{p+1}(t)} \int_{1-m / p+1}^{1-1 / p+1} u^{\alpha p}(1-u)^{\alpha-1} d u
\end{aligned}
$$

since for $p \geq 2 m,(1-m /(p+1))^{-p} \leq e^{2 m}$.

Finally,

$$
\begin{aligned}
J_{5} \leq & \beta_{1}\left(\frac{t}{(p+1) !}\right) \frac{(1+1 / p)^{\delta_{p+1}(t) p}}{(p+1)^{\delta_{p+1}}(t)} \int_{A_{5}} u^{\left(\alpha+\delta_{p+1}(t)\right) p}(1-u)^{\alpha-\delta_{p+1}(t)-1} d u \\
\leq & \beta_{1}\left(\frac{t}{(p+1) !}\right)\left(\frac{(1+1 / p)^{p}(1-1 /(m(p+1)))^{p} m(p+1)}{p+1}\right)^{\delta_{p+1}(t)} \\
& \times \int_{A_{5}} u^{\alpha p}(1-u)^{\alpha-1} d u \\
\leq & \beta_{1}\left(\frac{t}{(p+1) !}\right) e^{\delta_{p+1}(t)} m^{\delta_{p+1}(t)} \int_{1-1 / p+1}^{1-1 / m(p+1)} u^{\alpha p}(1-u)^{\alpha-1} d u .
\end{aligned}
$$

Since

$$
\begin{aligned}
I_{4}+I_{5} \leq & \beta_{1}(t /((p+1) !))^{p+1}\left((m \exp (2 m))^{\delta_{p+1}(t)}+K m^{-\alpha / 2}\right)^{p} e^{2 m \delta_{p+1}(t)} m^{\delta_{p+1}(t)} \\
& \times \int_{1-m / p+1}^{1-1 / m(p+1)} u^{\alpha p}(1-u)^{\alpha-1} d u \\
\leq & \beta_{1}\left(\frac{t}{(p+1) !}\right)^{p+1}\left((m \exp (2 m))^{\delta_{p+1}(t)}+K m^{-\alpha / 2}\right)^{p} e^{2 m \delta_{p+1}(t)} m^{\delta_{p+1}(t)} C_{p}(\alpha),
\end{aligned}
$$

it follows that

$$
\begin{aligned}
& \frac{M_{1}(p+1, t)}{h(t /(p+1))^{p+1}\left(M\left(2 p_{0}\right)^{2 \delta}\right)^{2 p_{0}}}=\frac{I_{1}+I_{2}+I_{3}+I_{4}+I_{5}}{C_{p}(\alpha)} \\
& \leq m^{-\alpha / 2}+\beta_{1}(t /((p+1) !))^{p}\left((m \exp (2 m))^{\delta_{p+1}(t)}+K m^{-\alpha / 2}\right)^{p} \\
& \quad \times\left[(K-2) m^{-\alpha / 2}+m^{-\alpha / 2}\right] \\
& \quad+\beta_{1}(t /((p+1) !))^{p+1}\left((m \exp (2 m))^{\delta_{p+1}(t)}+K m^{-\alpha / 2}\right)^{p} e^{2 m \delta_{p+1}(t)} m^{\delta_{p+1}(t)},
\end{aligned}
$$

proving the induction step, since $\beta_{1}(t /((p+1) !))\left((m \exp (2 m))^{\delta_{p+1}(t)}+K m^{-\alpha / 2}\right)>$ 1. $\square$

Lemma 2.7. There exists constants $K$ and $M>0$ such that for every $m>K^{1 / \alpha}$, there is a $\tau$ such that for every $p \geq 2 m$ and $t \geq \tau^{p}$

$$
M_{-1}(p, t) \geq\left(M(2 m)^{2 \delta}\right)^{-2 m}\left(m e^{2 m}\right)^{-\delta_{p}^{1}(t) p}\left(1-\frac{K}{m^{\alpha}}\right)^{p} \beta_{-1}(t /(m p))^{p} h(t / p)^{p},
$$

where $\delta_{p}^{1}(t)=\sup _{s \geq t / m p 2^{p}}|\varepsilon(s)|$. 
Proof. Define $K=D+1$ where $D$ is chosen so that $1 \leq D \alpha(p+1)^{\alpha} C_{p}(\alpha)$ for every $p \geq 0$. We shall prove the lemma by induction over $p \geq 2 m$ for every fixed $m>K^{1 / \alpha}$. Since $h(t)^{p} \geq p^{-\delta p} h(t / p)^{p}$, by Lemma 2.5 , there exist $t_{0}$ and $M>0$ such that the statement holds for $p=2 m$ and $t \geq t_{0}$. Set $\tau=2 t_{0}$. Now suppose that the statement holds for $p(\geq 2 m)$.

Write

$$
M(p, t)=\frac{M_{-1}(p, t)}{h(t / p)^{p}\left(M(2 m)^{2 \delta}\right)^{-2 m}} .
$$

Then

$$
\begin{aligned}
M(p+1, t) & \\
= & \frac{1}{C_{p}(\alpha)} \int_{0}^{1} u^{\alpha p}\left(\frac{h\left(\frac{u t}{p}\right)}{h\left(\frac{t}{p+1}\right)}\right)^{p}(1-u)^{\alpha-1} \frac{h((1-u) t)}{h\left(\frac{t}{p+1}\right)} \beta_{-1}((1-u) t) M(p, u t) d u \\
\geq & \frac{1}{C_{p}(\alpha)} \int_{1-m / p+1}^{1-1 / m(p+1)} u^{\alpha p}\left(\frac{h(u t / p)}{h\left(\frac{t}{p+1}\right)}\right)^{p}(1-u)^{\alpha-1} \\
& \times \frac{h((1-u) t)}{h\left(\frac{t}{p+1}\right)} \beta_{-1}((1-u) t) M(p, u t) d u .
\end{aligned}
$$

We estimate this latter integral by splitting it into the sum of two integrals

$$
I_{l}=\int_{A_{l}} u^{\alpha p}\left(\frac{h(u t / p)}{h\left(\frac{t}{p+1}\right)}\right)^{p}(1-u)^{\alpha-1} \frac{h((1-u) t)}{h\left(\frac{t}{p+1}\right)} \beta_{-1}((1-u) t) M(p, u t) d u
$$

$(l=1,2)$, where

$$
A_{1}=[1-m /(p+1), 1-1 /(p+1)]
$$

and

$$
A_{2}=(1-1 /(p+1), 1-1 /(m(p+1))]
$$

We use

$$
\begin{aligned}
& \frac{h((1-u) t)}{h(t /(p+1))} \geq \begin{cases}(p+1)^{\delta_{p+1}^{1}(t)}(1-u)^{\delta_{p+1}^{1}(t)}, & \text { if } 1-\frac{1}{m(p+1)} \geq u \geq 1-\frac{1}{p+1} \\
(p+1)^{-\delta_{p+1}^{1}(t)}(1-u)^{-\delta_{p+1}^{1}(t)}, & \text { if } 1-\frac{1}{p+1} \geq u,\end{cases} \\
& \frac{h(u t / p)}{h(t /(p+1))} \geq \begin{cases}(1+1 / p)^{\delta_{p+1}^{1}(t)} u^{\delta_{p+1}^{1}(t)}, & \text { if } 1-\frac{1}{p+1} \geq u \geq 1-\frac{m}{p+1}, \\
(1+1 / p)^{-\delta_{p+1}^{1}(t)} u^{-\delta_{p+1}^{1}(t)}, & \text { if } u \geq 1-\frac{1}{p+1} .\end{cases}
\end{aligned}
$$

Again, it is important for the estimations of $M(p, u t)$ and $\beta_{-1}((1-u) t)$ in the integrals to note that for $1-\frac{m}{p+1} \leq u \leq 1-\frac{1}{m(p+1)}$ we have that $\delta_{p}^{1}(u t) \leq \delta_{p+1}^{1}(t)$ and that $\beta_{-1}((1-u) t /(m p)) \geq \beta_{-1}(t /(m(p+1)))$, and also $t \geq \tau^{p+1} \Rightarrow u t \geq \tau^{p}$.

Now we set

$$
J_{l}=I_{l} \beta_{-1}(t /(m(p+1)))^{-p}\left(m e^{2 m}\right)^{\delta_{p+1}^{1}(t) p}\left(1-\frac{K}{m^{\alpha}}\right)^{-p},
$$


and, by the induction hypothesis,

$$
\begin{aligned}
J_{1} & \geq \beta_{-1}\left(\frac{t}{m(p+1)}\right)\left(\frac{(1+1 / p)^{p}}{p+1}\right)^{\delta_{p+1}^{1}(t)} \int_{A_{1}} u^{\left(\alpha+\delta_{p+1}^{1}(t)\right) p}(1-u)^{\alpha-\delta_{p+1}^{1}(t)-1} d u \\
& \geq \beta_{-1}\left(\frac{t}{m(p+1)}\right)\left(\frac{(1-m /(p+1))^{p}}{(m /(p+1))(p+1)}\right)^{\delta_{p+1}^{1}(t)} \int_{A_{1}} u^{\alpha p}(1-u)^{\alpha-1} d u \\
& \geq \beta_{-1}\left(\frac{t}{m(p+1)}\right)\left(m e^{2 m}\right)^{-\delta_{p+1}^{1}(t)} \int_{1-m / p+1}^{1-1 / p+1} u^{\alpha p}(1-u)^{\alpha-1} d u
\end{aligned}
$$

since $p \geq 2 m$.

Similarly,

$$
\begin{aligned}
J_{2} \geq & \beta_{-1}\left(\frac{t}{m(p+1)}\right)\left(\frac{p+1}{(1+1 / p)^{p}}\right)^{\delta_{p+1}^{1}(t)} \int_{A_{2}} u^{\left(\alpha-\delta_{p+1}^{1}(t)\right) p}(1-u)^{\alpha+\delta_{p+1}^{1}(t)-1} d u \\
\geq & \beta_{-1}\left(\frac{t}{m(p+1)}\right)\left(\frac{p+1}{(1+1 / p)^{p}(1-1 /(m(p+1)))^{p} m(p+1)}\right)^{\delta_{p+1}^{1}(t)} \\
& \times \int_{A_{2}} u^{\alpha p}(1-u)^{\alpha-1} d u \\
\geq & \beta_{-1}\left(\frac{t}{m(p+1)}\right)(m e)^{-\delta_{p+1}^{1}(t)} \int_{1-1 / p+1}^{1-1 / m(p+1)} u^{\alpha p}(1-u)^{\alpha-1} d u .
\end{aligned}
$$

Consequently,

$$
\begin{aligned}
I_{1}+I_{2} \geq & \beta_{-1}(t /(m(p+1)))^{p+1}\left(m e^{2 m}\right)^{-\delta_{p+1}^{1}(t) p}\left(1-\frac{K}{m^{\alpha}}\right)^{p} \\
& \times e^{-2 m \delta_{p+1}^{1}(t)} m^{-\delta_{p+1}^{1}(t)} \int_{1-m / p+1}^{1-1 / m(p+1)} u^{\alpha p}(1-u)^{\alpha-1} d u .
\end{aligned}
$$

In order to compare the estimation with $C_{p}(\alpha)$ observe that

$$
\begin{aligned}
\int_{0}^{1-m / p+1} u^{\alpha p}(1-u)^{\alpha-1} d u & \leq \frac{p+1}{m} \int_{0}^{1} u^{\alpha p}(1-u)^{\alpha} d u \\
& =\frac{(p+1) \alpha}{m(1+\alpha(p+1))} C_{p}(\alpha)
\end{aligned}
$$

and

$$
\begin{aligned}
\int_{1-1 / m(p+1)}^{1} u^{\alpha p}(1-u)^{\alpha-1} d u & =\int_{0}^{1 / m(p+1)}(1-u)^{\alpha p} u^{\alpha-1} d u \\
& \leq \alpha^{-1} m^{-\alpha}(p+1)^{-\alpha} \leq D m^{-\alpha} C_{p}(\alpha)
\end{aligned}
$$


where the constant $D$ is chosen so that $1 \leq D \alpha(p+1)^{\alpha} C_{p}(\alpha)$. It follows that

$$
\begin{aligned}
I_{1}+I_{2} \geq & \beta_{-1}(t /(m(p+1)))^{p+1}\left(m e^{2 m}\right)^{-\delta_{p+1}^{1}(t) p}\left(1-\frac{K}{m^{\alpha}}\right)^{p} \\
& \times e^{-2 m \delta_{p+1}^{1}(t)} m^{-\delta_{p+1}^{1}(t)} C_{p}(\alpha)\left(1-\frac{1}{m}-\frac{D}{m^{\alpha}}\right) \\
\geq & \beta_{-1}(t /(m(p+1)))^{p+1}\left(m e^{2 m}\right)^{-\delta_{p+1}^{1}(t)(p+1)}\left(1-\frac{K}{m^{\alpha}}\right)^{p+1} C_{p}(\alpha)
\end{aligned}
$$

and

$$
\begin{aligned}
M_{-1}(p+1, t) \geq & \left(M(2 m)^{2 \delta}\right)^{-2 m} \beta_{-1}(t /(m(p+1)))^{p+1} \\
& \times\left(m e^{2 m}\right)^{-\delta_{p+1}^{1}(t)(p+1)}\left(1-\frac{K}{m^{\alpha}}\right)^{p+1} h(t /(p+1))^{p+1} .
\end{aligned}
$$

This finishes the induction step.

Lemma 2.8. For all $\beta>1$ there exists $t_{\beta}$ such that for every $t \geq t_{\beta}$ and $t_{\beta} \leq p \leq L_{2}(t)^{2}$ there exist $\theta_{i}=\theta_{i}(p, t, \beta) \in[-1,1] \quad(i=0, \pm 1)$ such that

$$
A_{i}(p, t)=\beta^{\theta_{i} p}\left(\Gamma(1+\alpha) t^{\alpha} h(t / p)\right)^{p} \frac{1}{\Gamma(1+\alpha p)} .
$$

Proof. Let $\beta>1$ be given. The upper estimation follows from Lemma 2.6 and the definition of $M_{1}(p, t)$ : First choose $m$ large enough and then $t_{\beta}$ so large that for $t \geq t_{\beta}$

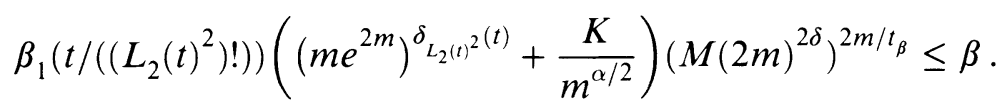

The proof for the lower estimation is similar using Lemma 2.7.

Lenima 2.9. For all $\beta>1$ there exists $t_{\beta}$ such that for all $n \geq t_{\beta}$ and $t_{\beta} \leq$ $p \leq L_{2}(n)^{2}$,

$$
\bar{a}(p, n)=\beta^{\theta p}\left(\Gamma(1+\alpha) n^{\alpha} h(n / p)\right)^{p} \frac{1}{\Gamma(1+\alpha p)}
$$

where $\theta=\theta(p, n) \in[-1,1]$. 
Proof. This follows immediately by Lemmas 2.3 and 2.8 .

Lemma 2.10. For all $\beta>1$ there exists $t_{\beta}$ such that for all $n \geq t_{\beta}$ and $t_{\beta} \leq$ $p \leq L_{2}(n)^{2}$

$$
\int_{A} S_{n}^{p} d \mu \geq \beta^{-p} \frac{p !}{\Gamma(1+\alpha p)}\left(\Gamma(1+\alpha) n^{\alpha} h(n / p)\right)^{p} .
$$

Proof. This follows from Lemmas 2.1 and 2.9 using the fact that $p !=\gamma_{p}(p)$.

Let

$$
f_{p}(x)=\sum_{q=1}^{p} \gamma_{p}(q) \frac{x^{p-q}}{\Gamma(1+\alpha q)} .
$$

Lemma 2.11. For all $\beta>1$ there exists $t_{\beta}$ such that for all $n \geq t_{\beta}$ and $t_{\beta} \leq$ $p \leq L_{2}(n)^{2}$

$$
\int_{A} S_{n}^{p} d \mu \leq \beta^{p}\left(\Gamma(1+\alpha) n^{\alpha} h(n / p)\right)^{p} f_{p}\left(\frac{e^{\delta}}{\beta \Gamma(1+\alpha) n^{\alpha} h(n / p)}\right) .
$$

Proof. By Lemma 2.1

$$
\int_{A} S_{n}^{p} d \mu=\sum_{q=1}^{p} \gamma_{p}(q) \bar{a}(q, n) .
$$

We shall use Lemma 2.9 for all $1 \leq p \leq L_{2}(n)^{2}$ multiplying by a suitable constant $M$ to compensate for low values of $p$. There is $t_{\beta}$ such that for $n \geq t_{\beta}$ and $1 \leq p \leq L_{2}(n)^{2}$

$$
\begin{aligned}
& \int_{A} S_{n}^{p} d \mu \leq M \sum_{q=1}^{p} \gamma_{p}(q) \frac{1}{\Gamma(1+\alpha q)}\left(\beta \Gamma(1+\alpha) n^{\alpha} h(n / q)\right)^{q} \\
& =M\left(\beta \Gamma(1+\alpha) n^{\alpha} h(n / p)\right)^{p} \sum_{q=1}^{p} \frac{\gamma_{p}(q)}{\Gamma(1+\alpha q)}\left(\frac{1}{\beta \Gamma(1+\alpha) n^{\alpha}}\right)^{p-q} \frac{h(n / q)^{q}}{h(n / p)^{p}} .
\end{aligned}
$$

Now $h\left((n / q)^{q} \leq(p / q)^{q \delta} h(n / p)^{q} \leq e^{\delta(p-q)} h(n / p)^{q}\right.$, whence

$$
\int_{A} S_{n}^{p} d \mu \leq M\left(\beta \Gamma(1+\alpha) n^{\alpha} h(n / p)\right)^{p} f_{p}\left(\frac{e^{\delta}}{\beta n^{\alpha} \Gamma(1+\alpha) h(n / p)}\right) .
$$

Lemma 2.12. There exists a constant $M$ such that for all $p$ and $y$

$$
f_{p}(y) \leq \frac{p ! M}{\Gamma(1+\alpha p)} e^{M p^{\alpha} y} .
$$

Proof. By definition of $\gamma_{p}(q)$ it follows that

$$
\begin{aligned}
f_{p+1}(y) & =\sum_{q=1}^{p+1} \gamma_{p+1}(q) \frac{y^{p+1-q}}{\Gamma(1+\alpha q)} \\
& =\sum_{q=1}^{p} \gamma_{p}(q) \frac{q y^{p+1-q}}{\Gamma(1+\alpha q)}+\sum_{q=1}^{p} \gamma_{p}(q) \frac{(q+1) y^{p-q}}{\Gamma(1+\alpha(q+1))} \\
& =\mathrm{A}+\mathrm{B} .
\end{aligned}
$$


Note that $\mathrm{A} \leq p y f_{p}(y)$. To estimate $\mathrm{B}$, we note that there exists $p_{0}$ such that for every $p \geq p_{0}$ and $1 \leq q \leq p$

$$
\frac{(q+1) \Gamma(1+\alpha q)}{\Gamma(1+\alpha(q+1))} \leq \frac{(p+1) \Gamma(1+\alpha p)}{\Gamma(1+\alpha(p+1))},
$$

(because the left-hand side is eventually increasing in $q$ ). Thus for $p \geq p_{0}$ we have

$$
B \leq(p+1) \frac{\Gamma(1+\alpha p)}{\Gamma(1+\alpha(p+1))} f_{p}(y)
$$

Hence for $p \geq p_{0}$

$$
\begin{aligned}
f_{p+1}(y) & \leq \frac{(p+1) \Gamma(1+\alpha p)}{\Gamma(1+\alpha(p+1))}\left(1+\frac{\Gamma(1+\alpha(p+1))}{\Gamma(1+\alpha p)} y\right) f_{p}(y) \\
& \leq \frac{(p+1) \Gamma(1+\alpha p)}{\Gamma(1+\alpha(p+1))}\left(1+M_{0} p^{\alpha} y\right) f_{p}(y)
\end{aligned}
$$

where $M_{0}>1$. Thus for $p \geq p_{0}$ and $y \geq 0$ and for some constant $M_{1}>0$

$$
\begin{aligned}
f_{p}(y) & \leq M_{1} \frac{p !}{\Gamma(1+\alpha p)} \prod_{k=1}^{p}\left(1+M_{0} k^{\alpha} y\right) f_{p_{0}}(y) \\
& \leq M_{1} \frac{p !}{\Gamma(1+\alpha p)} e^{M_{0} p^{\alpha+1} y} f_{p_{0}}(y) .
\end{aligned}
$$

Since $f_{q}\left(1 \leq q \leq p_{0}\right)$ are polynomials of degree $\leq p_{0}$ and $f_{q}(0)=1$ for all $q$, there is a constant $M_{2}$ such that $f_{q}(y) \leq e^{M_{2} y}$ for all $y>0$ and $q \leq p_{0}$, whence for all $y>0$ and $p \geq 1$

$$
f_{p}(y) \leq M_{1} \frac{p !}{\Gamma(1+\alpha p)} \exp \left(\left(M_{0} p^{\alpha+1}+M_{2}\right) y\right) .
$$

\section{Estimation OF PROBABILITIES}

Let $T$ be a conservative ergodic measure preserving transformation, $A$ a Darling-Kac set for $T$ of measure 1 and assume that $a_{n}(T) \sim n^{\alpha} h(n)$ where $h$ satisfies the assumptions of Theorem 2 and $0<\alpha<1$ (as before). In this section we estimate the probabilities $\mu\left(A \cap\left\{S_{n} \geq \frac{\Gamma(1+\alpha)}{\alpha^{\alpha}} n^{\alpha} h(n / t) t^{1-\alpha}\right\}\right)$ from above, and below, using Markov's inequality and Theorem 2. We prove

Theorem 3. For every $\beta>1$ there exists a constant $n_{\beta}$ such that for all $n_{\beta} \leq$ $t \leq L_{2}(n)^{2}$

$$
\begin{aligned}
\exp (-(1-\alpha) \beta t) & \leq \mu\left(A \cap\left\{S_{n} \geq \frac{\Gamma(1+\alpha)}{\alpha^{\alpha}} n^{\alpha} h(n / t) t^{1-\alpha}\right\}\right) \\
& \leq \exp (-(1-\alpha) t / \beta) .
\end{aligned}
$$

Remark. In case $T$ is a Markov shift, and $A$ is the event of being in a fixed state at a fixed time, the interarrival times to $A$ are i.i.d. random variables. 
This can be used to give a direct proof of theorem 3 by the method of Chernoff. See Lemma 3.1 of [25].

We first note that Theorem 2 is true for real $p$, by monotonicity of $\left(\int_{A} S_{n}^{p} d \mu\right)^{1 / p}$ in $p$ for fixed $n$. In this section we make use of the following notations:

$$
\begin{aligned}
& A(n, t)=A \cap\left\{S_{n} \geq \frac{\Gamma(1+\alpha)}{\alpha^{\alpha}} n^{\alpha} t^{1-\alpha} h(n / t)\right\}, \\
& B(n, t)=A \cap\left\{S_{n} \leq \frac{\Gamma(1+\alpha)}{\alpha^{\alpha}} n^{\alpha} t^{1-\alpha} h(n / t)\right\}, \\
& C(n, s, t)=A(n, s) \cap B(n, t) \\
& \quad=A \cap\left\{\frac{\Gamma(1+\alpha)}{\alpha^{\alpha}} n^{\alpha} s^{1-\alpha} h(n / s) \leq S_{n} \leq \frac{\Gamma(1+\alpha)}{\alpha^{\alpha}} n^{\alpha} t^{1-\alpha} h(n / t)\right\} .
\end{aligned}
$$

By the properties of $h$, the function $t^{1-\alpha} h(n / t)$ increases as $t$ increases, so that the interval defining $C(n, s, t)$ is not empty for $s<t$.

Lemma 3.1. For every $\beta>1$ there exist $n_{0}$ such that for all $n_{0} \leq t \leq L_{2}(n)^{2}$, $0 \leq p \leq t$.

$$
\int_{A(n, t)} S_{n}^{p} d \mu \leq\left(\frac{\Gamma(1+\alpha)}{\alpha^{\alpha}} n^{\alpha} h(n / t) t^{1-\alpha}\right)^{p} e^{(-(1-\alpha) t / \beta)} .
$$

Remark. Lemma 3.1 provides, in case $p=0$, the upper estimation in Theorem 3.

Proof. Let $n_{0}$ be as in Theorem 2 for $e^{\xi(1-\alpha)}$, where $\beta=(1-\xi)^{-1}$. Using Markov's inequality and Theorem 2, we obtain

$$
\begin{aligned}
\int_{A(n, t)} S_{n}^{p} d \mu & \leq\left(\frac{\Gamma(1+\alpha)}{\alpha^{\alpha}} n^{\alpha} h(n / t) t^{1-\alpha}\right)^{p-t} \int_{A} S_{n}^{t} d \mu \\
& \leq\left(\frac{\Gamma(1+\alpha)}{\alpha^{\alpha}} n^{\alpha} h(n / t) t^{1-\alpha}\right)^{p-t}\left(\frac{\Gamma(1+\alpha) e^{\xi(1-\alpha)}}{\alpha^{\alpha} e^{1-\alpha}} n^{\alpha} h(n / t) t^{1-\alpha}\right)^{t} \\
& \leq\left(\frac{\Gamma(1+\alpha)}{\alpha^{\alpha}} n^{\alpha} h(n / t) t^{1-\alpha}\right)^{p} e^{-(1-\alpha)(1-\xi) t} \cdot \square
\end{aligned}
$$

Lemma 3.2. For every $0<\xi<r<1$ and $\eta>0$ there exists $N$ such that for all $N \leq t \leq L_{2}(n)^{2}$ and $\xi t \leq p \leq r t$

$$
\int_{A(n, t)} S_{n}^{p} d \mu \leq \eta \int_{A} S_{n}^{p} d \mu
$$

Proof. For $0<r<1$ we have $1-\frac{1}{r}+\log \frac{1}{r}<0$. Choose $\beta_{0}>1$ such that $1-\frac{1}{\beta_{0} r}+\log \frac{1}{r}<0$, and then choose $\beta_{1}>1$ such that $1-\frac{1}{\beta_{0} r}+\log \frac{1}{r}+\log \beta_{1}^{2}<0$. This means that $a=\left(\beta_{1}^{2} e / r\right)^{(1-\alpha) r} e^{-(1-\alpha) \beta_{0}^{-1}}<1$. By Theorem 2 and Lemma 3.1 , there exists $N_{0}$ such that for all $N_{0} \leq p \leq t \leq L_{2}(n)^{2}$, we have

$$
\int_{A(n, t)} S_{n}^{p} d \mu \leq\left(\frac{\Gamma(1+\alpha)}{\alpha^{\alpha}} n^{\alpha} h(n / t) t^{1-\alpha}\right)^{p} e^{-(1-\alpha) t / \beta_{0}}
$$


and

$$
\int_{A} S_{n}^{p} d \mu \geq\left(\frac{\Gamma(1+\alpha)}{\alpha^{\alpha}} n^{\alpha} h(n / p) p^{1-\alpha}\right)^{p}
$$

whence

$$
\int_{A(n, t)} S_{n}^{p} d \mu \leq e^{-(1-\alpha) t / \beta_{0}}\left(\frac{\beta_{1} e t}{p}\right)^{(1-\alpha) p}\left(\frac{h(n / t)}{h(n / p)}\right)^{p} \int_{A} S_{n}^{p} d \mu .
$$

Since $h$ is slowly varying, there exists $N_{1} \geq N_{0}$ such that if $p, t \geq N_{1}$ and $\xi t \leq p \leq t \leq L_{2}(n)^{2}$ then

$$
\frac{h(n / t)}{h(n / p)} \leq \beta_{1}^{1-\alpha} .
$$

Thus for $p \geq N_{1}, \xi t \leq p \leq t \leq L_{2}(n)^{2}$

$$
\int_{A(n, t)} S_{n}^{p} d \mu \leq e^{-(1-\alpha) t / \beta_{0}}\left(\frac{\beta_{1}^{2} e t}{p}\right)^{(1-\alpha) p} \int_{A} S_{n}^{p} d \mu .
$$

It is not hard to check that the function $f(p)=\left(\beta_{1}^{2} e t p^{-1}\right)^{(1-\alpha) p}$ increases with $p$ when $p \in(0, t)$, whence for $p \geq N_{1}, \xi t \leq p \leq r t \leq L_{2}(n)^{2}$

$$
\begin{aligned}
\int_{A(n, t)} S_{n}^{p} d \mu & \leq e^{-(1-\alpha) t / \beta_{0}}\left(\frac{\beta_{1}^{2} e}{r}\right)^{(1-\alpha) r t} \int_{A} S_{n}^{p} d \mu \\
& =a^{t} \int_{A} S_{n}^{p} d \mu .
\end{aligned}
$$

By the choice of $\beta_{0}$ and $\beta_{1}, a<1$ and hence there exists $N \geq N_{1}$ such that $a^{t}<\eta$ for all $t \geq N$.

Lemma 3.3. For every $r<1, a<r / e$ and $\eta>0$ there exists $N$ such that

$$
\int_{B(n, a t)} S_{n}^{r t} d \mu \leq \eta \int_{A} S_{n}^{r t} d \mu
$$

for all $N \leq t \leq L_{2}(n)^{2}$.

Proof. First note that

$$
\int_{B(n, a t)} S_{n}^{r t} d \mu \leq\left(\frac{\Gamma(1+\alpha)}{\alpha^{\alpha}} n^{\alpha}(a t)^{1-\alpha} h(n / a t)\right)^{r t} .
$$

Choose $\beta>1$ such that $a<r e^{-1} \beta^{-2}$. By Theorem 2, there exists $N_{0}$ such that for every $N_{0} \leq t \leq L_{2}(n)^{2}$,

$$
\int_{A} S_{n}^{r t} d \mu \geq\left(\frac{\Gamma(1+\alpha)}{\beta^{1-\alpha} \alpha^{\alpha} e^{1-\alpha}} n^{\alpha}(r t)^{1-\alpha} h(n / r t)\right)^{r t},
$$

whence, for $N_{0} \leq t \leq L_{2}(n)^{2}$,

$$
\int_{B(n, a t)} S_{n}^{r t} d \mu \leq\left(\frac{\beta e a}{r}\right)^{(1-\alpha) r t}\left(\frac{h(n / a t)}{h(n / r t)}\right)^{r t} \int_{A} S_{n}^{r t} d \mu .
$$


Since $h$ is slowly varying, there exists $N_{1} \geq N$ such that for $N_{1} \leq t \leq L_{2}(n)^{2}$,

$$
\int_{B(n, a t)} S_{n}^{r t} d \mu \leq\left(\frac{\beta^{2} e a}{r}\right)^{(1-\alpha) r t} \int_{A} S_{n}^{r t} d \mu=b^{t} \int_{A} S_{n}^{r t} d \mu,
$$

where $b<1$ by the choice of $\beta$. Thus there exists $N \geq N_{1}$ such that

$$
\int_{B(n, a t)} S_{n}^{r t} d \mu \leq \eta \int_{A} S_{n}^{r t} d \mu
$$

whenever $N \leq t \leq L_{2}(n)^{2}$.

Lemma 3.4. For every $r<1, x<r, x<z<r \exp ((x / r)-1))$ and $\eta>0$ there exists $N$ such that for all $N \leq t \leq L_{2}(n)^{2}$

$$
\int_{C(n, x t, z t)} S_{n}^{r t} d \mu \leq \eta \int_{A} S_{n}^{r t} d \mu \text {. }
$$

Proof. There exists $\beta>1$ such that

$$
z<r \beta^{-2} \exp \left(x \beta^{-1} r^{-1}-1\right) \text {. }
$$

Then $a=\exp \left(x t \beta^{-1}\right)\left(\beta^{2} e z r^{-1}\right)^{r}<1$.

By Theorem 2 and Lemma 3.1 there exists $N_{0}$ such that for every $N_{0} \leq t \leq$ $L_{2}(n)^{2}$ we have $\mu(A(n, x t)) \leq \exp (-(1-\alpha) x t / \beta)$ and

$$
\int_{A} S_{n}^{r t} d \mu \geq\left(\frac{\Gamma(1+\alpha)}{\beta^{1-\alpha} \alpha^{\alpha} e^{1-\alpha}} n^{\alpha}(r t)^{1-\alpha} h(n / r t)\right)^{r t} .
$$

Since

$$
\int_{C(n, x t, z t)} S_{n}^{r t} d \mu \leq\left(\frac{\Gamma(1+\alpha)}{\alpha^{\alpha}} n^{\alpha}(z t)^{1-\alpha} h(n / z t)\right)^{r t} \mu(A(n, x t)),
$$

we have that for $N_{0} \leq t \leq L_{2}(n)^{2}$,

$$
\int_{C(n, x t, z t)} S_{n}^{r t} d \mu \leq e^{-(1-\alpha) x t / \beta}\left(\frac{\beta e z}{r}\right)^{(1-\alpha) r t}\left(\frac{h\left(\frac{n}{z t}\right)}{h\left(\frac{n}{r t}\right)}\right)^{r t} \int_{A} S_{n}^{r t} d \mu .
$$

Since $h$ is slowly varying, there exists $N_{1} \geq N_{0}$ such that for $N_{1} \leq t \leq L_{2}(n)^{2}$, $h(n /(z t))<\beta h(n /(r t))$, whence

$$
\int_{C(n, x t, z t)} S_{n}^{r t} d \mu \leq e^{-(1-\alpha) x t / \beta}\left(\frac{\beta^{2} e z}{r}\right)^{(1-\alpha) r t} \int_{A} S_{n}^{r t} d \mu=a^{(1-\alpha) t} \int_{A} S_{n}^{r t} d \mu .
$$

By the choice of $\beta, a<1$, whence there exists $N \geq N_{1}$ such that for every $N \leq t \leq L_{2}(n)^{2}$

$$
\int_{C(n, x t, z t)} S_{n}^{r t} d \mu \leq \eta \int_{A} S_{n}^{r t} d \mu
$$

Lemma 3.5. For every $r<1, z<r$ and $\eta>0$ there exists $N$ such that for all $N \leq t \leq L_{2}(n)^{2}$

$$
\int_{C(n, z t, t)} S_{n}^{r t} d \mu \geq(1-\eta) \int_{A} S_{n}^{r t} d \mu
$$


Proof. Let $\phi(x)=r \exp \left(x r^{-1}-1\right)$ for $x \in(0, r]$, and let $\phi^{1}=\phi$ and $\phi^{n+1}(x)=\phi\left(\phi^{n}(x)\right)$. Since $\phi(x) \leq x$ and $\phi(x)=x$ if and only if $x=r$, it follows that $\phi^{n}(x) \uparrow r$ as $n \uparrow \infty$.

Choose $a_{0}<r / e$, and $m \geq 1$ such that $\phi^{m}\left(a_{0}\right)>z$. By monotonicity and continuity of $\phi$ there are $a_{1}<a_{2}<\cdots<a_{m}$ such that $a_{m}>z$ and $a_{k+1}<\phi\left(a_{k}\right) \quad(0 \leq k<m)$.

By Lemmas 3.2, 3.3 and 3.4 there exists $N$ such that for every $N \leq t \leq$ $L_{2}(n)^{2}$,

$$
\begin{aligned}
& \int_{B(n, t)} S_{n}^{r t} d \mu \geq\left(1-\frac{\eta}{2}\right) \int_{A} S_{n}^{r t} d \mu, \\
& \int_{B\left(n, a_{0} t\right)} S_{n}^{r t} d \mu \leq \frac{\eta}{2(m+1)} \int_{A} S_{n}^{r t} d \mu, \\
& \int_{C\left(n, a_{k} t, a_{k+1} t\right)} S_{n}^{r t} d \mu \leq \frac{\eta}{2(m+1)} \int_{A} S_{n}^{r t} d \mu \quad(0 \leq k<m),
\end{aligned}
$$

whence

$$
\begin{aligned}
\int_{B(n, z t)} S_{n}^{r t} d \mu & \leq \int_{B\left(n, a_{m} t\right)} S_{n}^{r t} d \mu \\
& \leq \int_{B\left(n, a_{0} t\right)} S_{n}^{r t} d \mu+\sum_{k=0}^{m-1} \int_{C\left(n, a_{k} t, a_{k+1} t\right)} S_{n}^{r t} d \mu \\
& \leq \frac{\eta}{2} \int_{A} S_{n}^{r t} d \mu
\end{aligned}
$$

and

$$
\begin{aligned}
\int_{C(n, z t, t)} S_{n}^{r t} d \mu & \geq \int_{B(n, t)} S_{n}^{r t} d \mu-\int_{B(n, z t)} S_{n}^{r t} d \mu \\
& \geq(1-\eta) \int_{A} S_{n}^{r t} d \mu . \square
\end{aligned}
$$

Proof of Theorem 3. As remarked before, the upper estimation is proved in Lemma 3.1. We complete the proof by showing the lower estimation.

Suppose $z<1$ and let $z<r<1<\beta$. By Theorem 2 and Lemma 3.5 there exists $N$ such that for $N \leq t \leq L_{2}(n)^{2}$

$$
\int_{C(n, z t, t)} S_{n}^{r t} d \mu \geq\left(\frac{\Gamma(1+\alpha)}{\beta^{1-\alpha} \alpha^{\alpha} e^{1-\alpha}}(r t)^{1-\alpha} n^{\alpha} h(n /(r t))\right)^{r t}
$$

and $h(n /(r t)) \geq \beta^{-(1-\alpha)} h(n / t)$. Since

$$
\int_{C(n, z t, t)} S_{n}^{r t} d \mu \leq\left(\frac{\Gamma(1+\alpha)}{\alpha^{\alpha}} t^{1-\alpha} n^{\alpha} h(n / t)\right)^{r t} \mu(A(n, z t))
$$

we obtain that for $N \leq t \leq L_{2}(n)^{2}$,

$$
\mu(A(n, z t)) \geq\left(\frac{r}{\beta^{2} e}\right)^{(1-\alpha) r t}=e^{-(1-\alpha) t z \beta_{1}},
$$

where $\beta_{1}=\frac{r}{z}\left(1+\log \left(\frac{\beta^{2}}{r}\right)\right)$. 


\section{ASYMPTOTIC BEHAVIOUR OF ERGODIC SUMS}

In this section we suppose $T$ has a Darling-Kac set (as mentioned before, there is no loss of generality in assuming that it has measure 1), whose return time process is uniformly-or strongly mixing from below, and $a_{n}(T) \sim n^{\alpha} h(n)$ where $h(t)=C \exp \left[\int_{0}^{t} \varepsilon(s) / s d s\right]$ where

$$
\begin{aligned}
& \varepsilon(s)=0 \quad \forall 0<s<1, \\
& \lim _{s \rightarrow \infty} \varepsilon(s)=0, \\
& |\varepsilon(s)| \leq \delta<\frac{1}{4} \alpha \quad \forall s>0
\end{aligned}
$$

(as in the introduction).

Theorem 4. If $\phi(n) \uparrow$ and $\phi(n) / n \downarrow$ as $n \uparrow \infty$, then:

(a) If $\sum_{n=1}^{\infty} \frac{1}{n} \exp [-\beta \phi(n)]<\infty$ for all $\beta>1$ then

$$
\limsup _{n \rightarrow \infty} \frac{1}{n^{\alpha} h(n / \phi(n)) \phi(n)^{1-\alpha}} \sum_{k=1}^{n} f \circ T^{k} \leq K_{\alpha} \int_{X} f d \mu
$$

a.e. for every $f \in L_{+}^{1}$.

(b) If $\sum_{n=1}^{\infty} \frac{1}{n} \exp [-r \phi(n)]=\infty$ for all $r<1$ then

$$
\limsup _{n \rightarrow \infty} \frac{1}{n^{\alpha} h(n / \phi(n)) \phi(n)^{1-\alpha}} \sum_{k=1}^{n} f \circ T^{k} \geq K_{\alpha} \int_{X} f d \mu
$$

a.e. for every $f \in L_{+}^{1}$.

(c)

$$
\limsup _{n \rightarrow \infty} \frac{1}{n^{\alpha} h\left(n / L_{2}(n)\right) L_{2}(n)^{1-\alpha}} \sum_{k=1}^{n} f \circ T^{k}=K_{\alpha} \int_{X} f d \mu
$$

a.e. for every $f \in L_{+}^{1}$.

The proof of this theorem will be given at the end of the section.

Proposition 1. Suppose in addition to the above that

$$
\sup \left\{|\varepsilon(s)-\varepsilon(t)|: \frac{t}{L_{2}(t)} \leq s \leq t\right\}=o\left(\left(L_{3}(t)\right)^{-1}\right) \text { as } t \rightarrow \infty \text {. }
$$

If $\phi(n) \uparrow$ and $\phi(n) / n \downarrow$ as $n \uparrow \infty$, then:

(a) If $\sum_{n=1}^{\infty} \frac{1}{n} \exp \left[-(\beta \phi(n))^{(1-\alpha-\varepsilon(n))^{-1}}\right]<\infty$ for all $\beta>1$ then

$$
\limsup _{n \rightarrow \infty} \frac{1}{n^{\alpha} h(n) \phi(n)} \sum_{k=1}^{n} f \circ T^{k} \leq K_{\alpha} \int_{X} f d \mu
$$

a.e. for every $f \in L_{+}^{1}$.

(b) If $\sum_{n=1}^{\infty} \frac{1}{n} \exp \left[-(r \phi(n))^{(1-\alpha-\varepsilon(n))^{-1}}\right]=\infty$ for all $r<1$ then

$$
\limsup _{n \rightarrow \infty} \frac{1}{n^{\alpha} h(n) \phi(n)} \sum_{k=1}^{n} f \circ T^{k} \geq K_{\alpha} \int_{X} f d \mu
$$

a.e. for every $f \in L_{+}^{1}$. 
(c)

$$
\limsup _{n \rightarrow \infty} \frac{1}{n^{\alpha} h(n) L_{2}(n)^{1-\alpha-\varepsilon(n)}} \sum_{k=1}^{n} f \circ T^{k}=K_{\alpha} \int_{X} f d \mu
$$

a.e. for every $f \in L_{+}^{1}$.

Remark. For every $\beta>1, M<\infty$, there is an $N \ni \forall N \leq t \leq M L_{2}(n)$,

$$
h(n / t)=\beta^{\theta} t^{-\varepsilon(n)} h(n)
$$

for some $\theta \in[-1,1]$.

Proof. The proof of the proposition is a minor modification of the proof of Theorem 4, in view of the above remark.

Corollary 2. Suppose that

$$
\sup _{t \leq s \leq t L_{2}(t)}\left|\frac{h(s)}{h(t)}-1\right| \rightarrow 0 \quad \text { as } t \rightarrow \infty .
$$

If $\phi(n) \uparrow$ and $\phi(n) / n \downarrow$ as $n \uparrow \infty$, then:

(a) If $\sum_{n=1}^{\infty} \frac{1}{n} \exp [-\beta \phi(n)]<\infty$ for all $\beta>1$ then

$$
\limsup _{n \rightarrow \infty} \frac{1}{n^{\alpha} h(n) \phi(n)^{1-\alpha}} \sum_{k=1}^{n} f \circ T^{k} \leq K_{\alpha} \int_{X} f d \mu
$$

a.e. for every $f \in L_{+}^{1}$.

(b) If $\sum_{n=1}^{\infty} \frac{1}{n} \exp [-r \phi(n)]=\infty$ for all $r<1$ then

$$
\limsup _{n \rightarrow \infty} \frac{1}{n^{\alpha} h(n) \phi(n)^{1-\alpha}} \sum_{k=1}^{n} f \circ T^{k} \geq K_{\alpha} \int_{X} f d \mu
$$

a.e. for every $f \in L_{+}^{1}$.

(c)

$$
\limsup _{n \rightarrow \infty} \frac{1}{n^{\alpha} h(n) L_{2}(n)^{1-\alpha}} \sum_{k=1}^{n} f \circ T^{k}=K_{\alpha} \int_{X} f d \mu
$$

a.e. for every $f \in L_{+}^{1}$.

Proof. This follows from Proposition 1, as for every $\beta>1$ and $M<\infty$ there is an $N$ such that for every $N \leq t \leq M L_{2}(n)^{2}, h(n / t)=\beta^{\theta} h(n)$ for some $\theta \in[-1,1]$.

For the proof of the theorem we need two lemmas which we show first. Let $A$ be a Darling-Kac set for $T$ such that $\mu(A)=1$.

Lemma 4.1. If $\phi(n) \geq 0$ then

(a) If $\sum_{n=1}^{\infty} \frac{1}{n} \exp [-\beta \phi(n)]<\infty$ for all $\beta>1$ then

$$
\sum_{n=1}^{\infty} \frac{1}{n} \mu\left(A \cap\left\{S_{n} \geq \beta K_{\alpha} n^{\alpha} h(n / \phi(n)) \phi(n)^{1-\alpha}\right\}\right)<\infty
$$

for all $\beta>1$. 
(b) If $\sum_{n=1}^{\infty} \frac{1}{n} \exp [-r \phi(n)]=\infty$ for all $r<1$ then

$$
\sum_{n=1}^{\infty} \frac{1}{n} \mu\left(A \cap\left\{S_{n} \geq r K_{\alpha} n^{\alpha} h(n / \phi(n)) \phi(n)^{1-\alpha}\right\}\right)=\infty
$$

for all $r<1$.

Proof. (a) Suppose $\beta>1$ and

$$
\sum_{n=1}^{\infty} \frac{1}{n} \exp [-\beta \phi(n)]<\infty
$$

By Theorem 3, there exist $N_{0}$ and $M$ such that

$$
\mu\left(A \cap\left\{S_{n} \geq \beta K_{\alpha} n^{\alpha} h(n / \phi(n)) \phi(n)^{1-\alpha}\right\}\right) \leq M \exp [-(\sqrt{\beta} \phi(n))]
$$

for $n \geq N_{0}, \phi(n) \leq 2 L_{2}(n)$ and

$$
\mu\left(A \cap\left\{S_{n} \geq \beta K_{\alpha} n^{\alpha} h(n / \phi(n)) \phi(n)^{1-\alpha}\right\}\right) \leq M\left(L_{2}(n)\right)^{-2}
$$

for $n \geq N_{0}, \phi(n)>2 L_{2}(n)$. Thus

$$
\sum_{n=1}^{\infty} \frac{1}{n} \mu\left(A \cap\left\{S_{n} \geq \beta K_{\alpha} n^{\alpha} h(n / \phi(n)) \phi(n)^{1-\alpha}\right\}\right)<\infty .
$$

(b) Suppose $r<1$ and $\sum_{n=1}^{\infty} \frac{1}{n} \exp [-r \phi(n)]=\infty$. By Theorem 3 there exist $N_{0}$ and $M$ such that

$$
\mu\left(A \cap\left\{S_{n} \geq r K_{\alpha} n^{\alpha} h(n / \phi(n)) \phi(n)^{1-\alpha}\right\}\right) \geq M^{-1} \exp [-\sqrt{r} \phi(n)]
$$

for $n \geq N_{0}, \phi(n) \leq 2 r^{-\frac{1}{2}} L_{2}(n)$. Since

$$
\sum \frac{1}{n} \exp [-r \phi(n)]<\infty
$$

where the sum $\sum$ extends over all $n$ such that $\phi(n)>2 r^{-\frac{1}{2}} L_{2}(n)$ we have that

$$
\sum_{n=1}^{\infty} \mu\left(A \cap\left\{S_{n} \geq r K_{\alpha} n^{\alpha} h(n / \phi(n)) \phi(n)^{1-\alpha}\right\}\right) \geq M^{-1} \sum \exp [-\sqrt{r} \phi(n)]=\infty
$$

where the sum $\sum$ extends over all $n$ such that $\phi(n) \leq 2 r^{-\frac{1}{2}} L_{2}(n)$.

Lemma 4.2. Suppose that $\phi(n) \uparrow, \phi(n) / n \downarrow$ as $n \uparrow \infty$. Let $\gamma>1$ and $K_{n}=$ $\left[\gamma^{n}\right]$. Then

(a)

$$
\sum_{n=1}^{\infty} \frac{1}{n} \mu\left(A \cap\left\{S_{n} \geq \beta K_{\alpha} n^{\alpha} h(n / \phi(n)) \phi(n)^{1-\alpha}\right\}\right)<\infty
$$

for all $\beta>1$ implies

$$
\sum_{n=1}^{\infty} \mu\left(A \cap\left\{S_{K_{n}} \geq \beta \gamma^{1+\alpha} K_{\alpha} K_{n}^{\alpha} h\left(K_{n} / \phi\left(K_{n}\right)\right) \phi\left(K_{n}\right)^{1-\alpha}\right\}\right)<\infty
$$

for all $\beta>1$. 
(b)

$$
\sum_{n=1}^{\infty} \frac{1}{n} \mu\left(A \cap\left\{S_{n} \geq r K_{\alpha} n^{\alpha} h(n / \phi(n)) \phi(n)^{1-\alpha}\right\}\right)=\infty
$$

for all $r<1$ implies

$$
\sum_{n=1}^{\infty} \mu\left(A \cap\left\{S_{K_{n}} \geq r \gamma^{-(1+\alpha)} K_{\alpha} K_{n}^{\alpha} h\left(K_{n} / \phi\left(K_{n}\right)\right) \phi\left(K_{n}\right)^{1-\alpha}\right\}\right)=\infty
$$

for all $r<1$.

Proof. From the assumptions on $h$, we have that $n^{\alpha} h(n) \uparrow$ as $n \uparrow$, and, setting $A(n)=n^{\alpha} h(n / \phi(n)) \phi(n)^{1-\alpha}$, we have that

(i) $A(n) \uparrow, A(n) / n \downarrow$ as $n \uparrow$,

(ii) $\forall \lambda>1 \exists n_{0}$ such that $\forall n \geq n_{0} A\left(K_{n+1}\right) \leq \lambda \gamma^{1+\alpha} A\left(K_{n}\right)$.

The lemma follows now from these two statements via a condensation argument.

Proof of Theorem 4. Set $A(n)=K_{\alpha} n^{\alpha} h(n / \phi(n)) \phi(n)^{1-\alpha}$. By the assumptions on $h$ and $\phi, n^{\delta} h(n / \phi(n)) \phi(n)^{1-\alpha} \uparrow$ as $n \uparrow$, whence

$$
\begin{aligned}
A(m n) & =K_{\alpha} m^{\alpha-\delta} n^{\alpha-\delta}\left((m n)^{\delta} h(m n / \phi(m n)) \phi(m n)^{1-\alpha}\right) \\
& \geq K_{\alpha} m^{\alpha-\delta} n^{\alpha-\delta}\left(n^{\delta} h(n / \phi(n)) \phi(n)^{1-\alpha}\right) \\
& =m^{\alpha-\delta} A(n) .
\end{aligned}
$$

Thus for all $\gamma>1$ and $M>1$ there exists $m$ such that for every $n A\left(K_{n+m}\right) \geq$ $M A\left(K_{n}\right)$ and theorem 1 is applicable, where we set $K_{n}=K_{n}(\gamma)=\left[\gamma^{n}\right] \quad(n \geq 1)$.

(a) Suppose that

$$
\sum_{n=1}^{\infty} \frac{1}{n} \exp [-\beta \phi(n)]<\infty \text { for all } \beta>1 .
$$

By Lemmas 4.1(a) and 4.2(a)

$$
\sum_{n=1}^{\infty} \mu\left(A \cap\left\{S_{K_{n}} \geq \beta \gamma^{1+\alpha} A\left(K_{n}\right)\right\}\right)<\infty \quad(\forall \beta>1, \gamma>1),
$$

whence by Theorem $1 \lim \sup _{n \rightarrow \infty} S_{K_{n}(\gamma)} / A\left(K_{n}(\gamma)\right) \leq \gamma^{1+\alpha}$ a.e. on $A$ for all $\gamma>1$. Thus

$$
\limsup _{n \rightarrow \infty} \frac{S_{n}}{A(n)} \leq \gamma^{1+\alpha} \limsup _{n \rightarrow \infty} \frac{A\left(K_{n+1}\right)}{A\left(K_{n}\right)} \leq \gamma^{2(1+\alpha)+\delta}
$$

a.e. on $A$ for every $\gamma>1$ since $h(n)$ is slowly varying and $\phi(n) / n \downarrow$ as $n \uparrow$. By ergodicity and the Hopf ergodic theorem

$$
\limsup _{n \rightarrow \infty} \frac{1}{A(n)} \sum_{k=1}^{n} f \circ T^{k} \leq \int_{X} f d \mu
$$

a.e. for every $f \in L_{+}^{1}$. 
(b) Suppose that

$$
\sum_{n=1}^{\infty} \frac{1}{n} \exp [-r \phi(n)]=\infty \text { for all } r<1 .
$$

By Lemmas 4.1(b) and 4.2(b)

$$
\sum_{n=1}^{\infty} \mu\left(A \cap\left\{S_{K_{n}} \geq r \gamma^{-(1+\alpha)} A\left(K_{n}\right)\right\}\right)=\infty \quad(\forall r<1, \gamma>1)
$$

whence by Theorem $1 \lim \sup _{n \rightarrow \infty} S_{K_{n}(\gamma)} / A\left(K_{n}(\gamma)\right) \geq \gamma^{-(1+\alpha)}$ a.e. on $A$ for all $\gamma>1$. Thus

$$
\limsup _{n \rightarrow \infty} \frac{S_{n}}{A(n)} \geq \limsup _{n \rightarrow \infty} \frac{S_{K_{n}}}{A\left(K_{n}\right)} \geq \gamma^{-(1+\alpha)}
$$

a.e. on $A$ for every $\gamma>1$ and, by ergodicity and the Hopf ergodic theorem

$$
\limsup _{n \rightarrow \infty} \frac{1}{A(n)} \sum_{k=1}^{n} f \circ T^{k} \geq \int_{X} f d \mu
$$

a.e. for every $f \in L_{+}^{1}$.

(c) Let $\phi(n)=L_{2}(n)$. Then

$$
\sum_{n=1}^{\infty} \frac{1}{n} \exp [-y \phi(n)]<\infty \quad \text { iff } y>1
$$

For $\gamma>1$, by condensation,

$$
\sum_{n=1}^{\infty} \exp \left[-y \phi\left(K_{n}\right)\right]<\infty \quad \text { iff } y>1
$$

and by Theorem 3

$$
\sum_{n=1}^{\infty} \mu\left(A \cap\left\{S_{K_{n}} \geq y K_{\alpha} K_{n}^{\alpha} h\left(K_{n} / \phi\left(K_{n}\right)\right) \phi\left(K_{n}\right)^{1-\alpha}\right\}\right)<\infty
$$

if and only if $y>1$.

Hence by Theorem 1 limsup $\operatorname{sum}_{n \rightarrow \infty} S_{K_{n}} / A\left(K_{n}\right)=1$ a.e. on $A$ for every $\gamma>1$. This implies $\lim \sup _{n \rightarrow \infty} S_{n} /\left(n^{\alpha} h(n / \phi(n)) \phi(n)^{1-\alpha}\right)=K_{\alpha}$ a.e. on $A$ and, as before, by ergodicity and the Hopf ergodic theorem

$$
\limsup _{n \rightarrow \infty} \frac{1}{n^{\alpha} h\left(n / L_{2}(n)\right) L_{2}(n)^{1-\alpha}} \sum_{k=1}^{n} f \circ T^{k}=K_{\alpha} \int_{X} f d \mu
$$

a.e. for every $f \in L_{+}^{1}$. 
Example. (See [2, $§ 1$, Example 2] for more details.) Let $T$ be the one-sided shift of a conservative, ergodic, measure preserving Markov operator, which is recurrent in the sense of T. E. Harris. By a theorem of N. Jain [13] there are constants $a_{n}>0, n \geq 1$ such that

$$
\lim _{n \rightarrow \infty} \frac{1}{a_{n}} \sum_{k=1}^{n} \widehat{T}^{k} f=\int_{X} f d \mu \quad \text { a.e., }
$$

although it is not known whether $T$ has a D-K set. However, by a theorem of E. Nummelin [22] $T$ is similar (see [1]) to the shift of a Markov chain with discrete state space, necessarily with asymptotically proportional return sequences, say $S$. It follows that if $A(n)>0$,

$$
\limsup _{n \rightarrow \infty} \frac{1}{A(n)} \sum_{k=1}^{n} f \circ T^{k}=\int f d \mu_{T} \quad \text { a.e., } \forall f \in L_{+}^{1}\left(\mu_{T}\right)
$$

if and only if:

$$
\limsup _{n \rightarrow \infty} \frac{1}{A(n)} \sum_{k=1}^{n} g \circ S^{k}=c \int g d \mu_{S} \quad \text { a.e. } \forall g \in L_{+}^{1}\left(\mu_{S}\right),
$$

where $c=\lim _{n \rightarrow \infty} a_{n}(S) / a_{n}(T)$. Thus, if $a_{n}(T)$ is regularly varying with index $\alpha \in(0,1)$, then $T$ satisfies the conclusion of Theorem 4 .

\section{THE OTHER LAW OF THE ITERATED LOGARITHM}

In this section we prove the other law of the iterated logarithm for nonnegative continued fraction mixing processes in the domain of attraction of positive stable laws of fractional index. This includes the independent case. Other examples can be found in [5].

Definition. Let $\left(X_{k}\right)_{k=1}^{\infty}$ be a stationary process defined on the probability space $(A, \mathscr{A}, m)$. The process is called continued fraction mixing, if

$$
\psi(n):=\sup \left\{\left|\frac{m(B \cap C)}{m(B) m(C)}-1\right|\right\}
$$

tends to zero, and $\psi(1)<\infty$, where the supremum is taken over all $k \geq 1$, and over all sets $B \in \sigma\left(X_{i}: i \leq k\right)$ and $C \in \sigma\left(X_{i}: i \geq k+n\right)$.

Given a continued fraction mixing processes taking values in the natural numbers we construct a conservative, ergodic, measure preserving transformation, which has the given process as a return time process on a Darling-Kac set.

We may assume that $A$ is a sequence space equipped with the $m$-preserving transformation $S\left(y_{k}\right)_{k=1}^{\infty}=\left(y_{k+1}\right)_{k=1}^{\infty}$ so that $X_{1}(y)=y_{1}$, whence $X_{n}(y)=$ $X_{1}\left(S^{n-1} y\right)=y_{n}$, where $y=\left(y_{k}\right)_{k=1}^{\infty}$. It is well known that $S$ is ergodic. 
Now we let

$$
\begin{aligned}
& X=\left\{x=(y, n): 1 \leq n \leq X_{1}(y), y \in A\right\}, \\
& \mathscr{B}=\bigvee_{n=1}^{\infty} \mathscr{A} \cap\left\{X_{1} \geq n\right\} \times\{n\}, \\
& \mu(B \times\{n\})=m(B) \quad\left(B \in \mathscr{A} \cap\left\{X_{1} \geq n\right\}\right), \\
& T(y, n)= \begin{cases}(y, n+1), & \text { if } X_{1}(y) \geq n+1, \\
(S y, 1), & \text { if } X_{1}(y)=n .\end{cases}
\end{aligned}
$$

By Kakutani's theorem [16] $T$ is a conservative, ergodic, measure preserving transformation of the $\sigma$-finite measure space $(X, \mathscr{B}, \mu)$. By Kac's formula [15] $\mu(X)=E X_{1}$. Moreover, identifying $A$ with $A \times\{1\}, X_{1}$ is the first return time function $\varphi$ to $A$, and $S$ is the transformation $T_{A}$ induced by $T$ on $A$, as defined in section 1. So, if $x=(y, 1) \in A$ then $S(y)=T_{A}(x)=T^{\varphi(x)}(x)$, where $\varphi(x)=X_{1}(y)$.

It is shown in [4, pp. 1043-1044] that $A$ is a D-K set for $T$.

Let $\left(Z_{n}\right)_{n=1}^{\infty}$ be a continued fraction mixing process. If for some sequence $\left(b_{n}\right)_{n=1}^{\infty}$, the distribution of $b_{n}^{-1} \sum_{k=1}^{n} Z_{k}$ converges, then its limit must be stable and the sequence $\left(b_{n}\right)_{n=1}^{\infty}$ is regularly varying (see [12]). Moreover, if the index $\alpha$ of the stable distribution belongs to $(0,1)$, then $b_{n}^{-1} \sum_{k=1}^{n}\left(\left[Z_{k}\right]+1\right)$ converges to the same limit distribution, since $\sum_{k=1}^{n}\left(\left[Z_{k}\right]+1\right)-\sum_{k=1}^{n} Z_{k}=$ $O(n)=o\left(b_{n}\right)$ because $\left(b_{n}\right)_{n=1}^{\infty}$ is regularly varying of order $1 / \alpha$. Clearly, the process $X_{k}=\left[Z_{k}\right]+1$ is again a continued fraction mixing process, and, making the above construction to obtain the conservative, ergodic, measure preserving transformation, the distribution of $a(n)^{-1} S_{n}$ converges, where $S_{n}=$ $\sum_{k=1}^{n} 1_{A} \circ T^{k}$, considered as random variables on $A$, and where $a$ denotes the inverse function to $b$, the extension of $\left(b_{n}\right)_{n=1}^{\infty}$ to the real line. Recall that $a_{n}(T), n \geq 1$ denotes the return sequence for $T$. It is not hard to show that

$$
\sup _{n \geq 1} \int_{A}\left(\frac{S_{n}}{a_{n}(T)}\right)^{2} d \mu<\infty,
$$

whence [1] $a_{n}(T)^{-1} S_{n} \rightarrow 1$ weakly in $L^{2}(m)$, and (see [2]), $a_{n}(T)^{-1} S_{n} 1$. It now follows from [2, Proposition 3] that $a_{n}(T) \sim c a(n)$ for some constant $c$. Thus $a_{n}(T)$ is regularly varying with index $\alpha$.

By the asymptotic renewal equation [4], $\mu(\{\varphi \geq t\}) \sim c / a(t)$ as $t \rightarrow \infty$. This shows, together with [4, Theorem 1], that for the continued fraction mixing process $\left(Z_{n}\right)_{n=1}^{\infty}, b_{n}^{-1} \sum_{k=1}^{n} Z_{k}$ converges to a stable distribution with index $\alpha \in(0,1)$ if and only if $m\left(\left\{Z_{1} \geq t\right\}\right) \sim c / a(t)$ as $t \rightarrow \infty$, where $a$ is regularly varying with index $\alpha$ and $b_{n} \sim c a^{-1}(n)$. Indeed, more precisely, $\mu(\{\varphi \geq t\}) \sim(\Gamma(1+\alpha) \Gamma(1-\alpha) a(t))^{-1}$ as $t \rightarrow \infty$, where $a(n)=a_{n}(T)$.

We now state the result of this section.

Theorem 5. Suppose that $\left(X_{n}\right)_{n=1}^{\infty}$ is a nonnegative continued fraction mixing stationary process, and that $m\left(\left\{X_{1} \geq t\right\}\right)=(\Gamma(1-\alpha) \Gamma(1+\alpha) a(t))^{-1}$, where 
$a(t)$ is regularly varying with index $\alpha \in(0,1)$. Let $b$ be the inverse of $a$. Then for $\phi(n) \uparrow$ and $\phi(n) / n \downarrow$ as $n \uparrow \infty$, we have:

(a) If $\sum_{n=1}^{\infty} \frac{1}{n} \exp [-\beta \phi(n)]<\infty$ for all $\beta>1$ then

$$
\liminf _{n \rightarrow \infty} \frac{1}{b(n / \phi(n)) \phi(n)} \sum_{k=1}^{n} X_{k} \geq K_{\alpha}^{-1 / \alpha} \quad \text { a.e. }
$$

(b) If $\sum_{n=1}^{\infty} \frac{1}{n} \exp [-r \phi(n)]=\infty$ for all $r<1$ then

$$
\liminf _{n \rightarrow \infty} \frac{1}{b(n / \phi(n)) \phi(n)} \sum_{k=1}^{n} X_{k} \leq K_{\alpha}^{-1 / \alpha} \quad \text { a.e. }
$$

(c)

$$
\liminf _{n \rightarrow \infty} \frac{1}{b\left(n / L_{2}(n)\right) L_{2}(n)} \sum_{k=1}^{n} X_{k}=K_{\alpha}^{-1 / \alpha} \quad \text { a.e. }
$$

Corollary 3. Suppose that

$$
\sup _{t \leq s \leq t L_{2}(t)}\left|\frac{h(s)}{h(t)}-1\right| \rightarrow 0 \quad \text { as } t \rightarrow \infty .
$$

If $\phi(n) \uparrow$ and $\phi(n) / n \downarrow$ as $n \uparrow \infty$, then:

(a) If $\sum_{n=1}^{\infty} \frac{1}{n} \exp [-\beta \phi(n)]<\infty$ for all $\beta>1$ then

$$
\liminf _{n \rightarrow \infty} \frac{1}{b(n) \phi(n)^{1-1 / \alpha}} \sum_{k=1}^{n} X_{k} \geq K_{\alpha}^{-1 / \alpha}
$$

(b) If $\sum_{n=1}^{\infty} \frac{1}{n} \exp [-r \phi(n)]=\infty$ for all $r<1$ then

$$
\liminf _{n \rightarrow \infty} \frac{1}{b(n) \phi(n)^{1-1 / \alpha}} \sum_{k=1}^{n} X_{k} \leq K_{\alpha}^{-1 / \alpha} \quad \text { a.e. }
$$

(c)

$$
\liminf _{n \rightarrow \infty} \frac{1}{b(n) L_{2}(n)^{1-1 / \alpha}} \sum_{k=1}^{n} X_{k}=K_{\alpha}^{-1 / \alpha} \quad \text { a.e. }
$$

Remark. Theorem 5(c) shows that the results in the corollary are only valid, if $h\left(n L_{2}(n)\right) \sim h(n)$. In the case of independent, identically distributed random variables, and for certain slowly varying functions the corollary follows from a result obtained by $\mathbf{M}$. Lipschutz [20]. However, it does not follow from [20], e.g. in case $h(t)=\exp \left((\log t)^{\gamma}\right)$ for $\gamma \in(0,1)$.

As mentioned above, there is no loss of generality in assuming that $A$ is a Darling-Kac set for the c.e.m.p.t. $T$ on the $\sigma$-finite measure space $(X, \mathscr{B}, \mu)$ and that the return time process to $A$ is given by $\left(X_{k}\right)_{k=1}^{\infty}=\left(\varphi \circ T_{A}^{k-1}\right)_{k=1}^{\infty}$, and that $a_{n}(T)=a(n)$. 
Lemma 5.1. Suppose that $B(n) / n \uparrow \infty$, and $\limsup _{n \rightarrow \infty} B(n x) / B(n) \leq \rho(x)$ $(x>1)$, where $\rho(x) \rightarrow 1$ as $x \downarrow 1$. Then

$$
\limsup _{n \rightarrow \infty} \frac{S_{n}}{B^{-1}(n)} \geq 1 \text { a.e. on } A
$$

if and only if

$$
\liminf _{n \rightarrow \infty} \frac{\varphi_{n}}{B(n)} \leq 1 \text { a.e. on } A
$$

where $B^{-1}$ denotes the inverse function to $B$.

Proof. Suppose that $\limsup _{n \rightarrow \infty} S_{n} / B^{-1}(n) \geq 1$ a.e. on $A$. Then for any $r<1 \quad S_{n}>r B^{-1}(n)$ infinitely often a.e. implies $\varphi_{\left[r B^{-1}(n)\right]} \leq n$ infinitely often a.e. Set $m_{n}=\left[r B^{-1}(n)\right]>r^{2} B^{-1}(n)$ for $n$ large enough. Then for $n$ large $B\left(m(n) / r^{2}\right) \geq n$ and

$$
\liminf _{m \rightarrow \infty} \frac{\varphi_{m}}{B(m)} \leq \limsup _{m \rightarrow \infty} \frac{B\left(m r^{-2}\right)}{B(m)} \leq \rho\left(r^{-2}\right) \rightarrow 1
$$

as $r \rightarrow 1$.

Now, suppose $\liminf _{n \rightarrow \infty} \varphi_{n} / B(n) \leq 1$ a.e. Then for all $\beta>1 \quad \varphi_{n} \leq \beta B(n)$ infinitely often a.e. implies that $S_{[\beta B(n)]} \geq n$ infinitely often a.e. Set $m(n)=$ $[\beta B(n)]$, then $B^{-1}(m(n) / \beta) \leq n$ and

$$
S_{m} \geq B^{-1}\left(\frac{m}{\beta}\right) \geq \frac{B^{-1}(m)}{\beta} \quad \text { infinitely often a.e. }
$$

Proof of Theorem 5. We write $a_{n}(T)=a(n)=n^{\alpha} h(n)$, where $h$ is slowly varying and satisfies the assumptions stated in the introduction. Then $a(t)$ increases and $a(t) / t$ decreases as $t$ increases. We denote by $b$ the inverse function of $a$. Then $b(t) / t$ increases as $t$ increases and $b$ is regularly varying with index $1 / \alpha$.

Let $\phi$ be as in the statement of the theorem. Define

$$
B(n)=K_{\alpha}^{-1 / \alpha} b(n / \phi(n)) \phi(n) .
$$

Clearly, $B(n)$ increases with $n$ and we denote by $A$ the inverse of $B$.

We first show that $B$ satisfies the assumptions of Lemma 5.1, that is $B(n) / n$ increases with $n$ and $\limsup _{n \rightarrow \infty} B(n x) / B(n) \rightarrow 1$ as $x \downarrow 1$.

We have

$$
\frac{B(n)}{n}=K_{\alpha}^{-1 / \alpha} \frac{b(n / \phi(n))}{n / \phi(n)} \uparrow \quad \text { as } n \uparrow \text { since } \phi(n) / n \downarrow \text { as } n \uparrow .
$$

Also, for $x>1$,

$$
\frac{B(n x)}{B(n)}=\frac{b(n x / \phi(n x)) \phi(n x)}{b(n / \phi(n)) \phi(n)} \leq x \frac{b(n x / \phi(n))}{b(n / \phi(n))} \rightarrow x^{1+1 / \alpha} \quad \text { as } n \rightarrow \infty .
$$

Thus $B$ satisfies the assumptions of Lemma 5.1. 
Next, we set $\psi=\phi \circ A$. Observe that $\psi(n)$ increases and $\psi(n) / n$ decreases as $n$ increases, since $A(t) \uparrow$ and $A(t) / t \downarrow$ as $t \uparrow$.

Now we prove

$$
\sum_{n=1}^{\infty} \frac{1}{n} \exp [-\beta \phi(n)]<\infty \quad \forall \beta>1 \Leftrightarrow \sum_{n=1}^{\infty} \frac{1}{n} \exp [-\beta \psi(n)]<\infty \quad \forall \beta>1
$$

and

$$
\sum_{n=1}^{\infty} \frac{1}{n} \exp [-r \phi(n)]=\infty \quad \forall r<1 \Leftrightarrow \sum_{n=1}^{\infty} \frac{1}{n} \exp [-r \psi(n)]=\infty \quad \forall r<1
$$

We prove the first statement, using a condensation argument. The second one has a similar proof.

Set $k_{n}=B\left(2^{n}\right)$. Since $B$ satisfies the condition of Lemma $5.1, k_{n+1} \geq 2 k_{n}$ and for $n$ large $k_{n+1} \leq 2^{2+1 / \alpha} k_{n}$. Thus

$$
k_{n} \leq k_{n+1}-k_{n} \leq M k_{n} \text { for } M=2^{2+1 / \alpha}-1
$$

and, clearly $k_{n} \uparrow$.

We have for fixed $\beta>1$

$$
\begin{gathered}
\sum_{n=1}^{\infty} \frac{1}{n} \exp [-\beta \psi(n)]=\sum_{n=1}^{\infty} \frac{1}{n} \exp [-\beta \phi(A(n))] \\
=\sum_{\nu=1}^{\infty} \sum_{k_{\nu} \leq n<k_{\nu+1}} \frac{1}{n} \exp [-\beta \phi(A(n))]<\infty \\
\left.\Leftrightarrow \quad \text { by the properties of }\left\{k_{n}\right\}\right) \\
\sum_{\nu=1}^{\infty} \exp \left[-\beta \phi\left(A\left(k_{\nu}\right)\right)\right]=\sum_{\nu=1}^{\infty} \exp \left[-\beta \phi\left(2^{\nu}\right)\right]<\infty \\
\Leftrightarrow \sum_{n=1}^{\infty} \frac{1}{n} \exp [-\beta \phi(n)]<\infty
\end{gathered}
$$

We are now in a position to prove the statements. Since (c) follows immediately from (a) and (b), which themselves have similar proofs, we only prove (a). 
Assume $\sum_{n=1}^{\infty} \frac{1}{n} \exp [-\beta \phi(n)]<\infty$ for every $\beta>1$. Then, by the above,

$$
\sum_{n=1}^{\infty} \frac{1}{n} \exp [-\beta \psi(n)]<\infty
$$

for every $\beta>1$ and by Theorem 4(a)

$$
\limsup _{n \rightarrow \infty} \frac{1}{K_{\alpha} n^{\alpha} h(n / \psi(n)) \psi(n)^{1-\alpha}} \sum_{k=1}^{n} 1_{A} \circ T^{k} \leq 1 .
$$

Since

we have

$$
n=B(A(n))=K_{\alpha}^{-1 / \alpha} b(A(n) / \phi(A(n))) \phi(A(n))
$$

whence

$$
b(A(n) / \phi(A(n)))=K_{\alpha}^{1 / \alpha} n / \phi(A(n)),
$$

$$
\begin{aligned}
A(n) & =a\left(K_{\alpha}^{1 / \alpha} n / \phi(A(n))\right) \phi(A(n))=a\left(K_{\alpha}^{1 / \alpha} n / \psi(n)\right) \psi(n) \\
& \sim K_{\alpha} n^{\alpha} h(n / \psi(n)) \psi(n)^{1-\alpha},
\end{aligned}
$$

since $h$ is slowly varying.

By Lemma 5.1,

$$
\begin{aligned}
& \liminf _{n \rightarrow \infty} \frac{1}{b(n / \phi(n)) \phi(n)} \sum_{k=1}^{n} X_{k} \\
& \quad=\liminf _{n \rightarrow \infty} \frac{1}{b(n / \phi(n)) \phi(n)} \varphi_{n} \geq K_{\alpha}^{-1 / \alpha} \text { a.e. }
\end{aligned}
$$

Proof of Corollary 3. Let $\phi(n)$ be as in the corollary. Let $\psi(n)=\phi(n) \wedge L_{2}(n)^{2}$. By Theorem 5,

$$
\frac{1}{b\left(n / L_{2}(n)^{2}\right) L_{2}(n)^{2}} \sum_{k=1}^{n} X_{k} \rightarrow \infty \quad \text { a.e. }
$$

Hence, we have that

$$
\begin{aligned}
& \liminf _{n \rightarrow \infty} \frac{1}{b(n / \phi(n)) \phi(n)} \sum_{k=1}^{n} X_{k} \leq K_{\alpha}^{-1 / \alpha} \quad \text { a.e. } \Leftrightarrow \\
& \liminf _{n \rightarrow \infty} \frac{1}{b(n / \psi(n)) \psi(n)} \sum_{k=1}^{n} X_{k} \leq K_{\alpha}^{-1 / \alpha} \quad \text { a.e. }
\end{aligned}
$$

We now claim that

$$
b(n / \psi(n)) \psi(n) \sim b(n) \psi(n)^{1-1 / \alpha} .
$$

This is because, under the conditions imposed on $h$, for any $\beta>1$ and $n$ large enough and $L_{2}(n)^{-2} \leq t \leq L_{2}(n)^{2}, a\left(t^{1 / \alpha} n\right)=\beta^{\theta} t a(n)$ for some $-1 \leq \theta \leq 1$, whence $a\left(t^{1 / \alpha} b(n)\right)=\beta^{\theta}$ tn and so

$$
t^{1 / \alpha} b(n)=b\left(\beta^{\theta} t n\right)=\beta^{\theta^{\prime} / \alpha} b(t n)
$$

for some $\theta^{\prime} \in(-2,2)$. Putting $t=1 / \psi(n)$ establishes our claim. 
Noting that, in addition to the above, $\psi(n) \uparrow$ and $\psi(n) / n \downarrow$ as $n \uparrow$, and that

$$
\sum_{n=1}^{\infty} \frac{1}{n} \exp [-\beta \phi(n)]<\infty \leftrightarrow \sum_{n=1}^{\infty} \frac{1}{n} \exp [-\beta \psi(n)]<\infty,
$$

Corollary 3 follows easily from Theorem 5 .

\section{REFERENCES}

1. J. Aaronson, Rational ergodicity and a metric invariant for Markov shifts, Israel J. Math. 27 (1977), 93-123.

2. __ The asymptotic distributional behaviour of transformations preserving infinite measure, J. Analyse Math. 39 (1981), 203-234.

3. __ An ergodic theorem with large normalizing constants, Israel J. Math. 38 (1981), 182188.

4. __ Random f-expansions, Ann. Probab. 14 (1986), 1037-1057.

5. R. L. Adler, Continued fractions and Bernoulli trials, Ergodic Theory (J. Moser, E. Phillips and S. Varadhan, Eds.), Courant Inst. Math. Sci., New York, 1975.

6. R. L. Adler and B. Weiss, The ergodic infinite measure preserving transformation of Boole, Israel J. Math. 16 (1973), 263-278.

7. K. L. Chung and P. Erdös, On the applications of the Borel-Cantelli lemma, Trans. Amer. Math. Soc. 72 (1952), 179-186.

8. K. L. Chung and G. A. Hunt, On the zeroes of $\sum_{1}^{n} \pm 1$, Ann. of Math. (2) 50 (1949), 385-400.

9. D. A. Darling and M. Kac, On occupation times for Markov processes, Trans. Amer. Math. Soc. 84 (1957), 444-458.

10. M. D. Donsker and S. R. S. Varadhan, On laws of the iterated logarithm for local times, Comm. Pure Appl. Math. 30 (1977), 707-753.

11. E. Hopf, Ergodentheorie, Chelsea, New York, 1948.

12. I. B. Ibragimov and Y. V. Linnik, Independent and stationary sequences of random variables, Wolters-Noordhoff, Groningen, 1971.

13. N. C. Jain, Some limit theorems for general Markov processes, Z. Wahrsch. Verw. Gebiete 6 (1966), 206-233.

14. N. C. Jain and W. E. Pruitt, An invariance principle for the local time of a recurrent random walk, Z. Wahrsch. Verw. Gebiete 66 (1984), 141-156.

15. M. Kac, Of the notion of recurrence in discrete stochastic processes, Bull. Amer. Math. Soc. 53 (1947), 1002-1010.

16. S. Kakutani, Induced measure preserving transformations, Proc. Imp. Acad. Sci. Tokyo 19 (1943), 635-641.

17. H. Kesten, An iterated logarithm law for local time, Duke Math. J. 32 (1965), 447-456.

18. T. Li, F. Schweiger, The generalized Boole transformation is ergodic, Manuscripta Math. 25 (1978), 161-167.

19. M. Lipschutz, On strong laws for certain types of events connected with sums of independent random variables, Ann. of Math. 57 (1953), 318-330.

20. __ On strong bounds for sums of independent random variables which tend to a stable distribution, Trans. Amer. Math. Soc. 81 (1956), 135-154.

21. _ On the magnitude of the error in the approach of stable distributions. I, II, Indag. Math. 18 (1956), 281-287 and 288-294.

22. E. Nummelin, A splitting technique for Harris recurrent Markov chains, Z. Wahrsch. Verw. Gebiete 43 (1978), 309-318. 
23. E. Seneta, Regularly varying functions, Lecture Notes Math., vol. 508 Springer, Berlin, Heidelberg and New York, 1976.

24. M. Thaler, Transformations on $[0,1]$ with infinite invariant measures, Israel J. Math. 46 (1978), 233-253.

25. M. J. Wichura, Functional laws of the iterated logarithm for the partial sums of i.i.d. random variables in the domain of attraction of a completely asymmetric stable law, Ann. Probab. 6 (1974), 1108-1138.

School of Mathematical Sciences, Tel Aviv University, Ramat Aviv, 69978 Tel Aviv, ISRAEL

Institut für Mathematik Stochastik, Universität Göttingen, Lotzestr. 13, 3400 Göttingen, West Germany 